\title{
HOMOTOPY THEORY OF MINIMAL SIMPLICIAL SPACES
}

\author{
MARK D. PRITT
}

\begin{abstract}
Various aspects of homotopy theory in the category of minimal simplicial spaces are studied. It is shown that the usual results of homotopy theory hold in this category, and necessary and sufficient conditions are given under which a simplicial space has the homotopy type of a minimal simplicial space. Continuous cohomology in this category is also studied.
\end{abstract}

\section{INTRODUCTION}

Simplicial spaces are simplicial objects in the category of topological spaces. Many important topological spaces, such as the classifying space $B G$ of a topological group [7] and Haefliger's classifying space $B \Gamma_{q}$ for codimension $q$ foliations [5], are geometric realizations of simplicial spaces [8]. Unfortunately, the category of simplicial spaces is difficult to work with. For example, many of the results of homotopy theory do not hold in this category. In this paper we study minimal simplicial spaces and show that the subcategory of simplicial spaces having the homotopy type of minimal simplicial spaces is well behaved. In particular, the usual results of homotopy theory are valid in this subcategory. We also give necessary and sufficient conditions for a simplicial space to have the homotopy type of a minimal simplicial space, and we prove several theorems about the continuous cohomology of these simplicial spaces.

In the first three sections of this paper we present the main results, and we prove these results in the remaining four sections. As in [3], we shall be working implicitly in the category of compactly generated topological spaces [9]. We shall also assume for convenience that all simplicial spaces are connected; that is, given any two 0 -simplices $x, y \in X_{0}$ of the simplicial space $X$, there exists a 1-simplex $z \in X_{1}$ such that $\partial_{0} z=x$ and $\partial_{1} z=y$.

I would like to thank R. Szczarba for his guidance with this work and his patient assistance during the preparation of this paper.

\section{HOMOTOPY TYPE OF MINIMAL SIMPLICIAL SPACES}

In this section we study simplicial spaces which have the homotopy type of minimal simplicial spaces. We begin by defining a continuous version of the

Received by the editors August 21, 1989.

1980 Mathematics Subject Classification. Primary 55P15, 55U35; Secondary 55U10.

Key words and phrases. Simplicial space, minimal, continuous cohomology. 
Kan extension condition [6, Definition 1.3]. Let $X$ be a simplicial space. For $n \geq 1$ and $0 \leq k \leq n$ define

$$
X(n, k)=\left\{\left(x_{0}, \ldots, \widehat{x_{k}}, \ldots, x_{n}\right) \in\left(X_{n-1}\right)^{n} \mid \partial_{i} x_{j}=\partial_{j-1} x_{i}, i<j\right\},
$$

where $\left(X_{p}\right)^{q}=X_{p} \times \cdots \times X_{p}, q$ factors. There is a natural continuous mapping $\rho=\rho_{n, k}: X_{n} \rightarrow X(n, k)$ defined by $\rho(x)=\left(\partial_{0} x, \ldots, \widehat{\partial_{k} x}, \ldots, \partial_{n} x\right)$.

Definition 1.1. The simplicial space $X$ is a semi-Kan space if for every $n$ and $k, 0 \leq k \leq n$, there exists a continuous section $\lambda=\lambda_{n, k}: X(n, k) \rightarrow X_{n}$ of the mapping $\rho$ which satisfies the "degeneracy condition" $\lambda\left(\rho\left(s_{l} x\right)\right)=s_{l} x$ for $0 \leq l \leq n-1$ and $x \in X_{n-1}$.

The continuous sections $\lambda=\lambda_{n, k}$ in this definition are called the semi-Kan maps of $X$. It is an easy exercise, using the semi-Kan maps, to show that the homotopy groups of a semi-Kan space are topological groups.

Note that if we regard a semi-Kan space as a simplicial set (by "forgetting" the topology), then it satisfies the Kan extension condition [6, Definition 1.3]. We define a minimal, semi-Kan space to be a semi-Kan space which is minimal [6, Definition 9.1, Lemma 9.2] as a simplicial set; that is, homotopic simplices are equal.

Why do we use the term "semi-Kan" rather than "Kan"? Brown and Szczarba have given a definition of Kan simplicial spaces [3, Definition 2.1] which appears to be stronger than that of semi-Kan spaces. They chose their definition so that the function space $\mathfrak{F}(X, Y)$ is Kan whenever $Y$ is Kan [3, Theorem 2.17]. It turns out, however, that these two definitions are equivalent for minimal simplicial spaces (see the remark following Corollary 2.9).

Let $X$ be a semi-Kan space, and let $* \in X$ denote the degeneracies of a fixed basepoint $* \in X_{0}$. Define $X(n)$ and $\widetilde{X}_{n}$ by

$$
\begin{gathered}
X(n)=\left\{\left(x_{0}, \ldots, x_{n}\right) \in\left(X_{n-1}\right)^{n+1} \mid \exists x \in X_{n} \text { such that } \partial_{i} x=x_{i}\right\}, \\
\tilde{X}_{n}=\left\{x \in X_{n} \mid \partial_{i} x=*\right\} .
\end{gathered}
$$

Let $\rho=\rho_{n}: X_{n} \rightarrow X(n)$ denote the natural continuous surjective map defined by $\rho(x)=\left(\partial_{0} x, \ldots, \partial_{n} x\right)$, and let $\nu=\nu_{n}: \tilde{X}_{n} \rightarrow \pi_{n} X$ be the natural quotient map.

Theorem 1.2. Let $X$ be a semi-Kan space with basepoint *. Then $X$ contains a minimal, semi-Kan space as a strong deformation retract if and only if the following two conditions are satisfied:

(i) For every $n \geq 1$ there exists a continuous section $c=c_{n}: X(n) \rightarrow X_{n}$ of $\rho: X_{n} \rightarrow X(n)$ which satisfies the degeneracy condition $c\left(\rho\left(s_{k} x\right)\right)=s_{k} x$ for $0 \leq k \leq n-1$ and $x \in X_{n-1}$.

(ii) For every $n \geq 0$ there exists a continuous section $\alpha=\alpha_{n}: \pi_{n} X \rightarrow \tilde{X}_{n}$ of $\nu: \widetilde{X}_{n} \rightarrow \pi_{n} X$ satisfying the degeneracy condition $\alpha(0)=*$.

This theorem is a special case of Theorem 2.2. 
Definition 1.3. Let $f: X \rightarrow Y$ be a continuous simplicial map of semi-Kan spaces. Then $f$ is a strong equivalence if the induced homomorphism $f_{*}: \pi_{n} X$ $\rightarrow \pi_{n} Y$ is a homeomorphism for every $n$. In this case we say $X$ and $Y$ are strongly equivalent.

In particular, homotopy equivalent simplicial spaces are strongly equivalent.

Theorem 1.4. Let $f: X \rightarrow Y$ be a strong equivalence of minimal, semi-Kan spaces. Then $f$ is a simplicial homeomorphism.

The proof is given in $\S 5$. This theorem yields several interesting corollaries.

Corollary 1.5. Let $X$ be a semi-Kan space. Then $X$ contains a minimal, semiKan space as a strong deformation retract if and only if $X$ has the homotopy type of a minimal, semi-Kan space.

Proof. Suppose $X$ has the homotopy type of a minimal, semi-Kan space $M$. Then there are continuous simplicial maps $f: M \rightarrow X$ and $g: X \rightarrow M$ such that $f \circ g \simeq \mathrm{id}_{X}$ and $g \circ f \simeq \mathrm{id}_{M}$. By Theorem 1.4, $g \circ f$ is a simplicial homeomorphism. Let $M^{\prime}=f(M)$, and define $\tilde{f}=f \circ(g \circ f)^{-1}$. Then $\tilde{f}$ is a simplicial homeomorphism with inverse $\left.g\right|_{M^{\prime}}: M^{\prime} \rightarrow M$. Thus, $M^{\prime}$ is a minimal, semi-Kan space, and using the homotopy between $f \circ g$ and $\mathrm{id}_{X}$ one can check that $M^{\prime}$ is a strong deformation retract of $X$.

Definition 1.6. A homotopy-minimal space is a semi-Kan space which has the homotopy type of a minimal, semi-Kan space.

The following two corollaries of Theorem 1.4 are easy to prove.

Corollary 1.7. Let $X$ be a homotopy-minimal space, and let $M_{1}$ and $M_{2}$ be minimal, semi-Kan spaces, both of which are strong deformation retracts of $X$. Then $M_{1}$ and $M_{2}$ are simplicially homeomorphic.

Corollary 1.8. Two homotopy-minimal spaces $X_{1}$ and $X_{2}$ have the same homotopy type if and only if they are strongly equivalent.

For $\pi$ a topological abelian group, let $K(\pi, n)$ be the simplicial topological group defined by

$$
K(\pi, n)_{q}=Z^{n}(\Delta[q] ; \pi),
$$

the space of normalized cocycles, as in [6, pp. 100-101 or $3, \S 2$, p. 59]. For $\pi$ a nonabelian topological group, define $K(\pi, 1)$ by the usual bar construction [3, §2, Example 2].

Corollary 1.9. Let $n$ be a positive integer and $\pi$ a topological group (abelian if $n>1)$. Let $Z$ be a minimal, semi-Kan space such that $\pi_{n} Z=\pi$ and $\pi_{i} Z=0$, $i \neq n$. Then $Z$ is simplicially homeomorphic to $K(\pi, n)$.

Proof. Since $Z$ is minimal, $Z_{i}=*$ for $i<n$ and $Z_{n}=\pi$. Let $f: Z_{n} \rightarrow \pi$ be the identity map. By the addition theorem for simplicial sets [4, p. 19], $f$ is a cocycle (i.e., $\sum(-1)^{i} f\left(\partial_{i} z\right)=0$ for $\left.z \in Z_{n+1}\right)$. Thus, there exists a continuous 
simplicial mapping $f: Z \rightarrow K(\pi, n)$ (see [3, Lemma 3.5 or 6, Lemma 24.3]) which is a strong equivalence, hence a simplicial homeomorphism by Theorem 1.4.

Remark. Let $G$ be a simplicial topological group, and let $(\bar{G}, \partial)$ be its associated topological chain complex [6, Proposition 17.3], where $\bar{G}_{q}=G_{q} \cap$ $\operatorname{ker} \partial_{0} \cap \cdots \cap \operatorname{ker} \partial_{q-1}$ and $\partial=\partial_{q}: \bar{G}_{q} \rightarrow \bar{G}_{q-1}$. It can then be shown that $G$ is homotopy-minimal if and only if the chain complex $(\bar{G}, \partial)$ is splittable [3, Definition 8.2].

\section{Natural Postnikov systems of Minimal Simplicial SPACES}

To continue our study of minimal, semi-Kan spaces, we define semi-Kan fibrations and twisted products and use these mappings to define natural Postnikov systems. We first define semi-Kan fibrations. Let $p: E \rightarrow B$ be a continuous simplicial map of simplicial spaces, and for $n \geq 1$ and $0 \leq k \leq n$ define

$$
\begin{array}{r}
E(n, k)=\left\{\left(b ; e_{0}, \ldots, \widehat{e_{k}}, \ldots, e_{n}\right) \in B_{n} \times\left(E_{n-1}\right)^{n} \mid\right. \\
\left.\partial_{i} e_{j}=\partial_{j-1} e_{i} \text { for } i<j, \text { and } p e_{i}=\partial_{i} b\right\} .
\end{array}
$$

Then there is a natural continuous map $\rho=\rho_{n, k}: E_{n} \rightarrow E(n, k)$ defined by $\rho(e)=\left(p e ; \partial_{0} e, \ldots, \widehat{\partial_{k} e}, \ldots, \partial_{n} e\right)$.

Definition 2.1. The mapping $p: E \rightarrow B$ is a semi-Kan fibration if for every $n$ and $k, 0 \leq k \leq n$, there exists a continuous section $\lambda=\lambda_{n, k}$ of $\rho: E_{n} \rightarrow$ $E(n, k)$ which satisfies the degeneracy condition $\lambda\left(\rho\left(s_{l} e\right)\right)=s_{l} e$ for $0 \leq l \leq$ $n-1$ and $e \in E_{n-1}$.

The simplicial space $F=p^{-1}(*)$ is called the fiber of the semi-Kan fibration $p: E \rightarrow B$. We call the continuous sections $\lambda=\lambda_{n, k}$ the semi-Kan maps. Associated to any semi-Kan fibration is the usual exact sequence of homotopy groups with connecting homomorphisms induced from the semi-Kan maps (cf. [3, Theorem 6.5]).

Note that a semi-Kan fibration is a Kan fibration [6, Definition 7.1] as a map of simplicial sets. We define a minimal, semi-Kan fibration to be a semi-Kan fibration which is a minimal Kan fibration [6, Definition 10.1, Lemma 10.6] as a map of simplicial sets.

Let $p: E \rightarrow B$ be a semi-Kan fibration, let $E^{\prime}$ be a simplicial subspace of $E$, and define $p^{\prime}=\left.p\right|_{E^{\prime}}: E^{\prime} \rightarrow B$. We say $p^{\prime}: E^{\prime} \rightarrow B$ is a strong deformation retract of $p: E \rightarrow B$ if there is a continuous simplicial map $F: E \times \Delta[1] \rightarrow E$ defining a strong deformation retraction of $E^{\prime}$ in $E$ such that $p \circ F(x, u)=$ $p(x)$ for $(x, u) \in E_{q} \times \Delta[1]_{q}, q \geq 0$. Given a semi-Kan fibration $p: E \rightarrow B$, define

$$
\begin{aligned}
E(n)=\left\{\left(b ; e_{0}, \ldots, e_{n}\right) \in B_{n}\right. & \times\left(E_{n-1}\right)^{n+1} \mid \\
& \left.\exists e \in E_{n} \text { such that } p e=b \text { and } \partial_{i} e=e_{i}\right\},
\end{aligned}
$$


and let $\rho=\rho_{n}: E_{n} \rightarrow E(n)$ be the map defined by $\rho(e)=\left(p e ; \partial_{0} e, \ldots, \partial_{n} e\right)$. We can now give the following generalization of Theorem 1.2.

Theorem 2.2. Let $p: E \rightarrow B$ be a semi-Kan fibration with fiber $F$, and assume that $B_{0}=*$. Then $E$ contains a simplicial subspace $E^{\prime}$ such that the map $p^{\prime}=\left.p\right|_{E^{\prime}}: E^{\prime} \rightarrow B$ is a minimal, semi-Kan fibration and a strong deformation retract of $p: E \rightarrow B$ if and only if the following two conditions are satisfied:

(i) For every $n \geq 1$ there exists a continuous section $c=c_{n}: E(n) \rightarrow E_{n}$ of $\rho: E_{n} \rightarrow E(n)$ which satisfies the degeneracy condition $c\left(\rho\left(s_{k} e\right)\right)=s_{k} e$ for $0 \leq k \leq n-1$ and $e \in E_{n-1}$.

(ii) For every $n \geq 0$ there exists a continuous section $\alpha=\alpha_{n}: \pi_{n} F \rightarrow \widetilde{F}_{n}$ of the natural quotient map $\nu=\nu_{n}: \widetilde{F}_{n} \rightarrow \pi_{n} F$ which satisfies the degeneracy condition $\alpha(0)=*$.

The proof of this theorem is given in $\S 4$. Note that the hypothesis $B_{0}=*$ is satisfied when $B$ is minimal (and connected).

We now define twisted products. Let $B$ and $F$ be simplicial spaces, and let $\mathfrak{A}(F)$ be the simplicial space whose $n$-simplices are all continuous simplicial maps $f: F \times \Delta[n] \rightarrow F$ which are invertible (cf. [6, $\S 19$, p. 74]). There is a continuous simplicial action of $\mathfrak{A}(F)$ on $F$ given by $\alpha \cdot x=\alpha\left(x, \Delta_{n}\right)$ for $\alpha \in \mathfrak{A}(F)_{n}, \quad x \in F_{n}$. We define a twisting map to be a sequence $\tau=\tau_{n}: B_{n} \rightarrow$ $\mathfrak{A}(F)_{n-1}$ of continuous mappings which satisfy the usual twisting identities [6, Definition 18.3]. Note that $\mathfrak{A}(F)$ is not necessarily a simplicial topological group, since the inverse map $\alpha \rightarrow \alpha^{-1}$ may not be continuous.

Definition 2.3. The twisted product $E=B \times_{\tau} F$ is the simplicial space defined by $E_{n}=B_{n} \times F_{n}$, with face and degeneracy operators the product of those in $B$ with those in $F$, except that

$$
\partial_{0}(b, x)=\left(\partial_{0} b, \tau(b) \cdot \partial_{0} x\right)
$$

for $(b, x) \in B_{n} \times F_{n}$. We often refer to the map $p: E \rightarrow B$, defined by $p(b, x)=b$, as the twisted product, and we call $E$ the total space, $B$ the base space, and $F$ the fiber.

In Lemmas 2.4 and 2.6 we give conditions under which a twisted product is a twisted cartesian product (TCP) [3, $\S 6$, pp. 80-81].

Lemma 2.4. Let $E=B \times_{\tau} F$ be a twisted product. Suppose each topological space $F_{q}$ is locally compact and locally connected. Then $E$ is a TCP.

Proof. By [1, Theorem 4], the group of homeomorphisms of $F_{q}$ is a topological group. It is then easy to check that $\mathfrak{A}(F)$ is a simplicial topological group and $E$ is a TCP.

We now define principal twisted products. Let $B \times{ }_{\tau} G$ be a twisted product whose fiber $G$ is a simplicial topological group. There is a natural simplicial inclusion $G \hookrightarrow \mathfrak{A}(G)$ defined as follows. For $x \in G_{n}$, let $\bar{x}: \Delta[n] \rightarrow G$ be the 
natural simplicial map determined by $x$, and define the continuous simplicial $\operatorname{map} \theta_{x}: G \times \Delta[n] \rightarrow G$ by

$$
\theta_{x}(g, u)=g \cdot \bar{x}(u) \text { for }(g, u) \in G_{q} \times \Delta[n]_{q} .
$$

Then the mapping $x \mapsto \theta_{x}$ defines the inclusion of $G$ in $\mathfrak{A}(G)$.

Definition 2.5. Let $B \times{ }_{\tau} G$ be a twisted product whose fiber $G$ is a simplicial topological group. We define $B \times{ }_{\tau} G$ to be a principal twisted product if the image of the twisting map $\tau: B_{n} \rightarrow \mathfrak{A}(G)_{n-1}$ is contained in $G_{n-1}$ for every $n$.

A principal twisted cartesian product $(P T C P)$ is a TCP whose fiber and group are identical and in which the group acts on the fiber by left multiplication. The following lemma follows immediately from the definitions.

Lemma 2.6. Principal twisted products are PTCP's.

The proof of the following theorem is given in $\S 6$.

Theorem 2.7. A mapping $p: E \rightarrow B$, where $B_{0}=*$, is a minimal, semi-Kan fibration if and only if it is a twisted product with minimal, semi-Kan fiber.

We can now define natural Postnikov systems.

Theorem 2.8. Let $p: E=B \times{ }_{\tau} F \rightarrow B$ be a twisted product with minimal, semi-Kan fiber $F$, and assume $B_{0}=*$. Then there is a sequence

$$
\cdots \rightarrow E^{(n)} \rightarrow E^{(n-1)} \rightarrow \cdots \rightarrow E^{(1)} \rightarrow E^{(0)}=B
$$

of twisted products such that the fiber of $E^{(n)} \rightarrow E^{(n-1)}$ is $K\left(\pi_{n} F, n\right)$, and $E_{q}=E_{q}^{(n)}$ for $q \leq n$.

The proof is given in $\S 7$.

Corollary 2.9. Let $X$ be a minimal, semi-Kan space. Then there is a sequence

$$
\cdots \rightarrow X^{(n)} \rightarrow X^{(n-1)} \rightarrow \cdots \rightarrow X^{(1)} \rightarrow X^{(0)}=*
$$

of twisted products such that the fiber of $X^{(n)} \rightarrow X^{(n-1)}$ is $K\left(\pi_{n} X, n\right)$, and $X_{q}=X_{q}^{(n)}$ for $q \leq n$.

The sequence of twisted products appearing in this corollary is called the natural Postnikov system of $X$. It is a very useful tool for studying minimal, semi-Kan spaces. For example, using it and the easily demonstrated fact that any twisted product of Kan spaces is Kan, one can show that minimal, semiKan spaces are Kan (in the sense of [3]). In the next section we will use natural Postnikov systems to study continuous cohomology.

For $n>0$ and $\pi$ a topological group (abelian if $n>1$ ), let $\delta: L(\pi, n) \rightarrow$ $K(\pi, n)$ be the "universal fibration over $K(\pi, n)$ " [6, p. 102]. For a PTCP $E=K(G, n) \times{ }_{\tau} K(H, n-1)$ there exists a continuous simplicial mapping $f: K(G, n) \rightarrow K(H, n)$ such that $E$ is the pullback by $f$ of the universal fibration over $K(H, n)$ [6, Theorem 23.10]. Let $\widehat{f}: G \rightarrow H$ be the homomorphism induced by $f$ [6, Lemma 25.1]. 
Proposition 2.10. $E$ is homotopy-minimal if and only if for every $n$ there exist continuous (not necessarily homomorphic) sections of the natural epimorphisms

$$
\widehat{f}: G \rightarrow \operatorname{im} \widehat{f} \text { and } H \rightarrow \operatorname{coker} \widehat{f} .
$$

The proof of this proposition (which we leave to the reader) is an application of Theorem 1.2 and the fact that $E$ is the pullback by $f$ of the universal fibration over $K(H, n)$. We now give several examples of simplicial spaces which are not homotopy-minimal.

Example 2.11. Let $f: K(\mathbb{R}, n) \rightarrow K\left(S^{1}, n\right)$ be the simplicial mapping induced by the canonical epimorphism $\mathbb{R} \rightarrow S^{1}$, and let $E$ be the pullback by $f$ of the universal fibration over $K\left(S^{1}, n\right)$. The nontrivial connecting homomorphism in the exact homotopy sequence of the PTCP $E=K(\mathbb{R}, n) \times{ }_{\tau} K\left(S^{1}, n-1\right)$ is the canonical epimorphism $\mathbb{R} \rightarrow S^{1}$. Thus, $E$ has the homotopy groups of $K(\mathbb{Z}, n)$, but not its homotopy type by Proposition 2.10 .

Example 2.12. We now construct a noncontractible simplicial space whose homotopy groups are trivial. Let $\mathbb{R}^{\delta}$ denote the additive group of reals with its discrete topology, and let $k: K\left(\mathbb{R}^{\delta}, n\right) \rightarrow K(\mathbb{R}, n)$ be the simplicial mapping induced by the natural homomorphism $\mathbb{R}^{\delta} \rightarrow \mathbb{R}$. Define $U$ to be the pullback by $k$ of the universal fibration over $K(\mathbb{R}, n)$. Then all of the homotopy groups of $U$ are trivial, but $U$ is noncontractible by Proposition 2.10.

Example 2.13. We give another construction of a noncontractible simplicial space whose homotopy groups are trivial. Let $g: K(\mathbb{Z}, n+1) \rightarrow K(\mathbb{R}, n+1)$ be the simplicial map induced by the natural inclusion $\mathbb{Z} \hookrightarrow \mathbb{R}$, and define $V$ to be the pullback by $g$ of the universal fibration over $K(\mathbb{R}, n+1)$. Define $h: V \rightarrow L\left(S^{1}, n+1\right)$ to be the composition $V \rightarrow L(\mathbb{R}, n+1) \rightarrow L\left(S^{1}, n+1\right)$ of natural projections. Since the composition

$$
K(\mathbb{Z}, n+1) \stackrel{g}{\longrightarrow} K(\mathbb{R}, n+1) \stackrel{f}{\longrightarrow} K\left(S^{1}, n+1\right)
$$

is the zero map, where $f$ is the mapping defined in Example 2.11, the image of $h$ is contained in $K\left(S^{1}, n\right)$. Define $W$ to be the pullback by $h: V \rightarrow K\left(S^{1}, n\right)$ of the universal fibration over $K\left(S^{1}, n\right)$. Then examination of the exact homotopy sequence of $W$ yields $\pi_{i} W=0$ for all $i$, but $W$ is noncontractible as one can check using Theorem 1.2.

\section{Continuous COHOMOLOGY OF MiNimal SIMPLiCiAL SPACES}

We now use natural Postnikov systems (Corollary 2.9) and the Serre spectral sequence in continuous cohomology $[3, \S 8]$ to study the continuous cohomology of minimal, semi-Kan spaces. We first give an analogue of the Whitehead theorem. We next prove that if the homotopy groups of a minimal, semi-Kan space are compact Lie groups, then its continuous cohomology is trivial. We finally turn our attention to simplicial manifolds and smooth cohomology and prove 
that the smooth and continuous cohomologies of certain simplicial manifolds are isomorphic.

Definition 3.1. A homotopy-minimal space $X$ is simple if all of the twisted products in its natural Postnikov system are principal.

It can be shown that the twisted products in the natural Postnikov system of a homotopy-minimal space $X$ define a natural action of $\pi_{1} X$ on $\pi_{n} X$ for every $n$, and it can also be shown that $X$ is simple if and only if all of these actions are trivial (cf. [6, Theorem 25.7]).

We now give an analogue of the Whitehead theorem for simple spaces whose homotopy groups are abelian Lie groups. Define the dual homotopy groups $\left[3, \S 2\right.$, p. 63] of $X$ by $\pi^{n} X=\operatorname{Hom}_{\text {cont }}\left(\pi_{n} X, \mathbb{R}\right)$, and let $H^{n} X$ denote its continuous cohomology $[3, \S 2$, p. 58].

Theorem 3.2. Let $f: X \rightarrow Y$ be a continuous simplicial map of simple spaces, and assume $\pi_{n} X$ and $\pi_{n} Y$ are abelian Lie groups for all $n$. Then $f^{*}: H^{n} Y \rightarrow$ $H^{n} X$ is an isomorphism for every $n$ if and only if $f^{*}: \pi^{n} Y \rightarrow \pi^{n} X$ is an isomorphism for every $n$.

The proof is given at the end of this section.

The following theorem is a generalization of a classical result [10, Theorem 2] for compact Lie groups.

Theorem 3.3. Let $X$ be a homotopy-minimal space, all of whose homotopy groups are compact Lie groups. Then $H^{*}(X)$ is trivial.

Proof. Let $X^{(n)}$ be the $n$th space in the natural Postnikov system of $X$. Since the homotopy groups of $X$ are Lie groups, the twisted products $X^{(n)}=$ $X^{(n-1)} \times_{\tau_{n}} K\left(\pi_{n} X, n\right)$ are TCP's by Lemma 2.4. Furthermore, it is not difficult to show that the continuous cochain complex $C^{*}\left(K\left(\pi_{n} X, n\right)\right)$ is splittable [3, Definition 8.2], so we can apply the Serre spectral sequence [3, Theorem 8.3] to these TCP's.

We will show that $H^{*}\left(X^{(n)}\right)$ is trivial for all $n$. For $n=1$, we have $X^{(1)}=K\left(\pi_{1} X, 1\right)$. Since $H^{*}\left(K\left(\pi_{1} X, 1\right)\right)$ is the continuous group cohomology of the compact Lie group $\pi_{1} X$, it is trivial by the Van Est Theorem [10, Theorem 2]. As an inductive step, assume $H^{*}\left(X^{(n-1)}\right)$ is trivial. Then, applying an inductive argument using the Van Est Theorem and the Serre spectral sequence to the universal fibration over $K\left(\pi_{n} X, q\right)$ for every $q \leq n$, it can be shown that the continuous cohomology of $K\left(\pi_{n} X, n\right)$ is trivial. Application of the Serre spectral sequence to the TCP $X^{(n)}=X^{(n-1)} \times_{\tau_{n}} K\left(\pi_{n} X, n\right)$ then yields $H^{*}\left(X^{(n)}\right)=H^{*}\left(X^{(n-1)}\right)$.

We now turn our attention to simplicial manifolds and smooth cohomology. Our basic result is that for certain simplicial manifolds, the smooth and continuous cohomologies are isomorphic. 
Definition 3.4. A simplicial space $X$ is a simplicial manifold if each $X_{n}$ is a (finite-dimensional) smooth manifold, and if the face and degeneracy maps are smooth maps. A semi-Kan simplicial manifold is a simplicial manifold $X$ which is also a semi-Kan space with smooth semi-Kan maps.

Definition 3.5. For a simplicial manifold $X$ and locally convex topological vector space $V$, define the smooth cohomology of $X$ with coefficients in $V$, $H_{s}^{*}(X ; V)$, to be the homology of the cochain subcomplex $C_{s}^{*}(X ; V)$ of the continuous cochain complex $C^{*}(X ; V)$, where $C_{s}^{n}(X ; V)$ is the space of all smooth mappings $f: X_{n} \rightarrow V$ which vanish on degeneracies.

We call $H_{s}^{*} X=H_{s}^{*}(X ; \mathbb{R})$ the smooth cohomology of $X$. Note that, for any Lie group $G$, the smooth cohomology of $K(G, 1)$ is by definition the same as the smooth group cohomology of $G$. Note also that there is a natural homomorphism $H_{s}^{*} X \rightarrow H^{*} X$ induced by the inclusion $C_{s}^{*}(X) \hookrightarrow C^{*}(X)$.

Definition 3.6. A PTCP $E=B \times{ }_{\tau} G$ is a smooth PTCP if $B$ is a simplicial manifold, $G$ is a simplicial Lie group, and the twisting map $\tau=\tau_{n}: B_{n} \rightarrow G_{n-1}$ is smooth for every $n$.

It is easy to check that if $E=B \times{ }_{\tau} G$ is a smooth PTCP, then $E$ is a simplicial manifold.

Theorem 3.7. For any smooth PTCP $E=B \times{ }_{\tau} G$, there exists a spectral sequence $\left\{\mathscr{E}_{r}^{p, q}\right\}$ converging to $H_{s}^{*}(E)$ such that

$$
\mathscr{E}_{2}^{p, q}=H_{s}^{p}\left(B ; H_{s}^{q}(G)\right)
$$

Proof. The proof is exactly analogous to that of [3, Theorem 8.3]. (Cf. $\S \S I X .5 .9$ and IX.6.1.5 of [2].) The only difference occurs in showing

$$
C_{s}^{p}\left(B ; H_{s}^{q}(G)\right) \cong \frac{\operatorname{ker} \delta^{\prime}: C_{s}^{p}\left(B ; C_{s}^{q}(G)\right) \rightarrow C_{s}^{p}\left(B ; C_{s}^{q+1}(G)\right)}{\operatorname{im} \delta^{\prime}: C_{s}^{p}\left(B ; C_{s}^{q-1}(G)\right) \rightarrow C_{s}^{p}\left(B ; C_{s}^{q}(G)\right)}
$$

Since $B_{p}$ is a manifold, $C_{s}^{p}(B, \cdot)$ is an exact functor from the category of Fréchet spaces to Fréchet spaces [2, §IX.6.1.5]. Applying it to the short exact sequences of Fréchet spaces

$$
\begin{gathered}
0 \rightarrow B_{s}^{q}(G) \hookrightarrow Z_{s}^{q}(G) \rightarrow H_{s}^{q}(G) \rightarrow 0, \\
0 \rightarrow Z_{s}^{q}(G) \hookrightarrow C_{s}^{q}(G) \stackrel{\delta}{\rightarrow} B_{s}^{q+1}(G) \rightarrow 0,
\end{gathered}
$$

yields (3.8).

We call $\left\{\mathscr{E}_{r}^{p, q}\right\}$ the Serre spectral sequence in smooth cohomology.

Theorem 3.9. Let $X$ be a semi-Kan simplicial manifold which is simple and whose homotopy groups are Lie groups. Then the natural homomorphism $H_{s}^{*} X$ $\rightarrow H^{*} X$ is an isomorphism.

Proof. Let $X^{(n)}$ be the $n$th space in the natural Postnikov system of $X$. Since $K\left(\pi_{n} X, n\right)$ is a simplicial Lie group for every $n$ and since the semi-Kan maps 
of $X$ are smooth, $X^{(n)}=X^{(n-1)} \times_{\tau_{n}} K\left(\pi_{n} X, n\right)$ is a smooth PTCP for every $n$. Thus, $X^{(n)}$ is a simplicial manifold.

To prove the theorem, it suffices to show that the map $H_{s}^{*}\left(X^{(n)}\right) \rightarrow H^{*}\left(X^{(n)}\right)$ is an isomorphism for every $n$. For $n=1$ this is the well-known result which gives an isomorphism between the smooth and continuous cohomologies of a Lie group. As an inductive step, assume it is true for $n-1$. Let $\left\{\mathscr{E}_{r}^{p, q}\right\}$ be the Serre spectral sequence in smooth cohomology, and $\left\{E_{r}^{p, q}\right\}$ the Serre spectral sequence in continuous cohomology, for the smooth PTCP $X^{(n)}=$ $X^{(n-1)} \times_{\tau_{n}} K\left(\pi_{n} X, n\right)$. There is a natural homomorphism $\left\{\mathscr{E}_{r}^{p, q}\right\} \rightarrow\left\{E_{r}^{p, q}\right\}$ of spectral sequences induced by the inclusion $C_{s}^{*}\left(X^{(n)}\right) \hookrightarrow C^{*}\left(X^{(n)}\right)$. The natural homomorphism

$$
\mathscr{E}_{2}^{p, 0}=H_{s}^{p}\left(X^{(n-1)}\right) \rightarrow H^{p}\left(X^{(n-1)}\right)=E_{2}^{p, 0}
$$

is an isomorphism for all $p$ by the inductive step. Similarly, the homomorphism

$$
\mathscr{E}_{2}^{0, q}=H_{s}^{q}\left(K\left(\pi_{n} X, n\right)\right) \rightarrow H^{q}\left(K\left(\pi_{n} X, n\right)\right)=E_{2}^{0, q}
$$

is an isomorphism for all $q$ by Lemma 3.10 below. Since $E_{2}^{0, q}$ and $\mathscr{E}_{2}^{0, q}$ are finite-dimensional as real vector spaces, the homomorphism

$$
\mathscr{E}_{2}^{p, q}=\mathscr{E}_{2}^{p, 0} \otimes \mathscr{E}_{2}^{0, q} \rightarrow E_{2}^{p, 0} \otimes E_{2}^{0, q}=E_{2}^{p, q}
$$

is then an isomorphism for all $p$ and $q$. This gives an isomorphism of spectral sequences, whence $H_{s}^{*}\left(X^{(n)}\right) \rightarrow H^{*}\left(X^{(n)}\right)$ is an isomorphism.

Lemma 3.10. If $G$ is a Lie group, then for every $q$ the natural homomorphism

$$
H_{s}^{q}(K(G, n)) \rightarrow H^{q}(K(G, n))
$$

is an isomorphism of finite-dimensional real vector spaces.

Proof. For $n=1$, the isomorphism follows from the well-known result for Lie groups, and the finite-dimensionality follows from the Van Est Theorem [10, Theorem 2]. For $n>1, G$ must be abelian, and it is not difficult to show that $G$ must then be of the form $G=\mathbb{R}^{k} \times\left(S^{1}\right)^{m} \times \mathbb{Z}^{l} \times H$, where $H$ is any finite abelian group. The lemma then follows by applying the usual Serre spectral sequence argument [3, Lemma 10.1$]$ to the universal fibration over $K(G, q)$ for every $q$.

Proof of Theorem 3.2. We will need the following result. In [3, $\S 2$, p. 61] there is defined a contravariant functor $\Delta$ from the category of topological DG algebras to that of simplicial spaces; $\Delta(A)$ is the "simplicial realization" of the algebra $A$. In [3, Theorems 2.11 and 2.13] it is proven essentially that, given a simple space $X$ whose homotopy groups are abelian Lie groups, there exists an algebra $M$ and a continuous simplicial map $f: X \rightarrow \Delta(M)$ which induces isomorphisms in continuous cohomology and dual homotopy groups. Furthermore, given another algebra $M^{\prime}$ and a map $f^{\prime}: X \rightarrow \Delta\left(M^{\prime}\right)$ which induces an 
isomorphism in continuous cohomology, there exists an algebra isomorphism $h: M^{\prime} \rightarrow M$ such that the maps $\Delta(h) \circ f$ and $f^{\prime}$ are homotopic.

Suppose $f^{*}: H^{*} Y \rightarrow H^{*} X$ is an isomorphism. Choose algebras $M$ and $N$, and continuous simplicial maps $\alpha: X \rightarrow \Delta(M)$ and $\beta: Y \rightarrow \Delta(N)$, as above. Since $\beta \circ f: X \rightarrow \Delta(N)$ induces an isomorphism on continuous cohomology, there exists an algebra isomorphism $h: N \rightarrow M$ such that $\Delta(h) \circ \alpha \simeq \beta \circ f$. That is, the following diagram commutes up to homotopy:

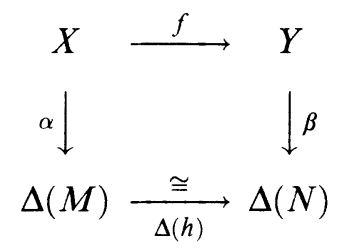

Applying the dual homotopy functor $\pi^{n}(\cdot)$ to this diagram for every $n$ yields the fact that $f^{*}: \pi^{n} Y \rightarrow \pi^{n} X$ is an isomorphism for every $n$.

Conversely, suppose $f$ induces an isomorphism on dual homotopy groups. Let $X^{(n)}$ and $Y^{(n)}$ be the $n$th spaces in the natural Postnikov systems of $X$ and $Y$, respectively, and note that $f$ induces a map $f: X^{(n)} \rightarrow Y^{(n)}$. We will prove by induction that $f^{*}: H^{*}\left(Y^{(n)}\right) \rightarrow H^{*}\left(X^{(n)}\right)$ is an isomorphism for every $n$. For $n=1$ this is true since $H^{q}\left(K\left(\pi_{1} X, 1\right)\right)$ is equal to zero for $q>1$ and $\pi^{1} X$ for $q=1$. As an inductive step, assume $f^{*}: H^{*}\left(Y^{(n-1)}\right) \rightarrow H^{*}\left(X^{(n-1)}\right)$ is an isomorphism. Then the map of PTCP's induced by $f$,

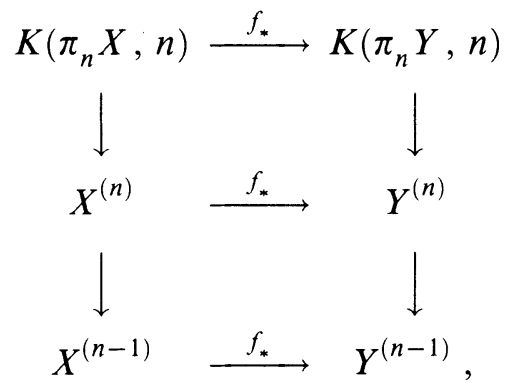

induces isomorphisms in continuous cohomology on the fibers and the bases. Since $H^{q}\left(K\left(\pi_{n} X, n\right)\right)$ is finite-dimensional by Lemma 3.10, the $E_{2}$ terms of the Serre spectral sequences for the two PTCP's simplify to $E_{2}^{p, q}=E_{2}^{p, 0} \otimes E_{2}^{0, q}$. Thus, $f$ induces an isomorphism in continuous cohomology on the total spaces.

\section{Proof of Theorem 2.2}

Let $p: E \rightarrow B$ be a semi-Kan fibration with fiber $F$, and suppose $E$ contains a simplicial subspace $E^{\prime}$ such that the map $p^{\prime}=\left.p\right|_{E^{\prime}}: E^{\prime} \rightarrow B$ is a minimal, semi-Kan fibration and strong deformation retract of $p: E \rightarrow B$. We must show that for every $n$ there exist continuous sections $\alpha=\alpha_{n}: \pi_{n} F \rightarrow \widetilde{F}_{n}$ and $c=c_{n}: E(n) \rightarrow E_{n}$ of the natural mappings $\nu=\nu_{n}: \widetilde{F}_{n} \rightarrow \pi_{n} F$ and $\rho=$ $\rho_{n}: E_{n} \rightarrow E(n)$, respectively, which satisfy the degeneracy conditions $\alpha(0)=*$ 
and $c\left(\rho\left(s_{k} e\right)\right)=s_{k} e$. Let $F^{\prime}$ be the fiber of the minimal, semi-Kan fibration $p^{\prime}: E^{\prime} \rightarrow B$. Since $F^{\prime}$ is a strong deformation retract of $F$ and since $\widetilde{F_{n}^{\prime}}=$ $\left\{x \in F_{n}^{\prime} \mid \partial_{i} x=*\right\}=\pi_{n}\left(F^{\prime}\right)$, the continuous section $\alpha: \pi_{n} F \rightarrow \widetilde{F}_{n}$ is given essentially by the inclusion of ${\widetilde{F_{n}}}_{n}^{\prime}$ in $\widetilde{F}_{n}$.

We now show that a continuous section $c: E(n) \rightarrow E_{n}$ of the natural map $\rho: E_{n} \rightarrow E(n)$ exists and satisfies the degeneracy condition. This is done in two steps and is an immediate consequence of the following two lemmas.

Let $p: E \rightarrow B$ be as above, and define spaces $H_{n}$ and $H(n)$ by

$$
\begin{gathered}
H_{n}=\left\{z \in E_{n} \mid \exists x \in E_{n-1} \text { with } \partial_{i} z=\partial_{i} s_{n-1} x \text { for } i \leq n-1\right. \\
\left.\quad \text { and } p z=s_{n-1} p x\right\}, \\
H(n)=\left\{(x, y) \in E_{n-1} \times E_{n-1} \mid x \stackrel{(p)}{\sim} y\right\} .
\end{gathered}
$$

Let $\zeta=\zeta_{n}: H_{n} \rightarrow H(n)$ be the natural continuous map defined by $\zeta(z)=$ $\left(\partial_{n-1} z, \partial_{n} z\right)$. Note that $\zeta$ is surjective.

Lemma 4.1. Let $p: E \rightarrow B$ and $\zeta=\zeta_{n}: H_{n} \rightarrow H(n)$ be as above. Then there exists a continuous section $h=h_{n}: H(n) \rightarrow H_{n}$ of $\zeta: H_{n} \rightarrow H(n)$ which satisfies the degeneracy condition $h(x, x)=s_{n-1} x$ for $x \in E_{n-1}$.

Proof. For $n \geq 0$ and $0 \leq i \leq n$, let $h_{i}: E_{n} \rightarrow E_{n+1}$ be a collection of "homotopy maps" defining the strong deformation retraction of $p^{\prime}: E^{\prime} \rightarrow B$ in $p: E \rightarrow B$. That is, the $h_{i}$ 's satisfy the conditions $\partial_{0} h_{0}=\mathrm{id}_{E_{n}}, \partial_{n+1} h_{n} e \in E_{n}^{\prime}$ for $e \in E_{n}, p \circ h_{i}=s_{i} \circ p$, and $h_{i} e^{\prime}=s_{i} e^{\prime}$ for $e^{\prime} \in E_{n}^{\prime}$, as well as the usual conditions [6, Definition 5.1]. We will use the $h_{i}$ 's to define the section $h$.

Let $(x, y) \in H(n)$. For $n=1$, define $h(x, y)=\partial_{0} \lambda\left(s_{1} s_{0} p x ;-, h_{0} x, h_{0} y\right)$. For $n>1$, define $\omega_{i} \in E_{n}$ by induction from $i=n-1$ to $i=0$ as follows. Let

$$
\begin{aligned}
\omega_{n-1}=\partial_{n-1} \lambda\left(s_{n} s_{n-1} p x ; \partial_{0} s_{n} h_{n-1} x, \ldots,\right. \\
\left.\partial_{n-2} s_{n} h_{n-1} x,-, h_{n-1} x, h_{n-1} y\right) \in E_{n} .
\end{aligned}
$$

Then $w_{n-1}: \partial_{n-1} h_{n-1} x \stackrel{(p)}{\sim} \partial_{n-1} h_{n-1} y$. For the inductive step, let $0<r<n-1$ and assume $\omega_{r+1} \in E_{n}$ is defined so that $\omega_{r+1}: \partial_{r+1} h_{r+1} x \stackrel{(p)}{\sim} \partial_{r+1} h_{r+1} y$. Define

$$
\begin{aligned}
\omega_{r}=\partial_{r} \lambda\left(s_{n} s_{r} p x ; \partial_{0} s_{n} h_{r} x, \ldots, \partial_{r-1} s_{n} h_{r} x,-, \omega_{r+1},\right. \\
\left.\partial_{r+2} s_{n} h_{r} x, \ldots, \partial_{n-1} s_{n} h_{r} x, h_{r} x, h_{r} y\right) \in E_{n} .
\end{aligned}
$$

Then $\omega_{r}: \partial_{r} h_{r} x \stackrel{(p)}{\sim} \partial_{r} h_{r} y$. For $r=0$ we get $\omega_{0}: x \stackrel{(p)}{\sim} y$, so define $h(x, y)=$ $\omega_{0}$. It is straightforward to show that $h$ satisfies the degeneracy condition.

Lemma 4.2. There is a continuous section $h=h_{n}: H(n) \rightarrow H_{n}$ of the map $\zeta: H_{n} \rightarrow H(n)$ which satisfies the degeneracy condition $h(x, x)=s_{n-1} x$ for $x \in E_{n-1}$ if and only if there is a continuous section $c=c_{n}: E(n) \rightarrow E_{n}$ of the 
map $\rho: E_{n} \rightarrow E(n)$ which satisfies the degeneracy condition $c\left(\rho\left(s_{k} e\right)\right)=s_{k} e$ for $0 \leq k \leq n-1$ and $e \in E_{n-1}$.

The proof of this lemma is straightforward, so we leave it to the reader.

To complete the proof of Theorem 2.2, let $p: E \rightarrow B$ be a semi-Kan fibration with fiber $F$ such that $B_{0}=*$. Suppose for every $n$ there exist continuous sections $\alpha=\alpha_{n}: \pi_{n} F \rightarrow \widetilde{F}_{n}$ and $c=c_{n}: E(n) \rightarrow E_{n}$ of the natural mappings $\nu=\nu_{n}: \widetilde{F}_{n} \rightarrow \pi_{n} F$ and $\rho=\rho_{n}: E_{n} \rightarrow E(n)$, respectively, which satisfy the degeneracy conditions $\alpha(0)=*$ and $c\left(\rho\left(s_{k} e\right)\right)=s_{k} e$. We must construct a simplicial subspace $E^{\prime}$ of $E$ such that the map $p^{\prime}=\left.p\right|_{E^{\prime}}: E^{\prime} \rightarrow B$ is a minimal, semi-Kan fibration and strong deformation retract of $p: E \rightarrow B$. To construct $E^{\prime}$, we will need continuous sections $\beta=\beta_{n}: E_{n} / \stackrel{(p)}{\sim} \rightarrow E_{n}$ of the natural quotient mappings $q=q_{n}: E_{n} \rightarrow E_{n} / \stackrel{(p)}{\sim}$.

Proposition 4.3. Let $p: E \rightarrow B$ be a semi-Kan fibration. Suppose for every $n$ there exist continuous sections $\alpha=\alpha_{n}: \pi_{n} F \rightarrow \widetilde{F}_{n}$ and $c=c_{n}: E(n) \rightarrow E_{n}$ as above. Then for every $n$ there exists a continuous section $\beta=\beta_{n}: E_{n} / \stackrel{(p)}{\rightarrow} \rightarrow E_{n}$ of the natural quotient map $q=q_{n}: E_{n} \rightarrow E_{n} / \stackrel{(p)}{\sim}$ which satisfies the degeneracy condition $\beta\left(q\left(s_{k} e\right)\right)=s_{k}$ e for $e \in E_{n-1}$ and $0 \leq k \leq n-1$.

The proof of this proposition is given at the end of the section.

We can now define the simplicial subspace $E^{\prime}$ of $E$. Let $\varepsilon_{n}: E_{n} \rightarrow E_{n}$ be the continuous mapping defined by $\varepsilon_{n}=\beta_{n} \circ q_{n}$. Let $E_{0}^{\prime}=*$. Assuming $E_{n-1}^{\prime}$ is defined, let $Y_{n}=\left\{x \in E_{n} \mid \partial_{i} x \in E_{n-1}^{\prime}\right\}$, and define $E_{n}^{\prime}=\varepsilon_{n}\left(Y_{n}\right)$. It is easy to check that $E^{\prime}$ is a well-defined simplicial subspace of $E$. Define $p^{\prime}=\left.p\right|_{E^{\prime}}: E^{\prime} \rightarrow B$.

Proposition 4.4. $p^{\prime}: E^{\prime} \rightarrow B$ is a strong deformation retract of $p: E \rightarrow B$.

Proof. For $n \geq 0$ and $0 \leq i \leq n$ we must define a collection of "homotopy maps" $h_{i}: E_{n} \rightarrow E_{n+1}$ which satisfy the conditions $\partial_{0} h_{0}=\mathrm{id}_{E_{n}}, \partial_{n+1} h_{n} e \in E_{n}^{\prime}$ for $e \in E_{n}, p \circ h_{i}=s_{i} \circ p$, and $h_{i} e^{\prime}=s_{i} e^{\prime}$ for $e^{\prime} \in E_{n}^{\prime}$, as well as the usual conditions [6, Definition 5.1]. The definition of the $h_{i}$ 's follows from standard simplicial techniques using the mappings $\varepsilon_{n}: E_{n} \rightarrow E_{n}$ and $c=c_{n}: E(n) \rightarrow E_{n}$. We leave the details to the reader.

To complete the proof of Theorem 2.2, we need only show that $p^{\prime}: E^{\prime} \rightarrow B$ is a minimal, semi-Kan fibration. Since any retract of a semi-Kan fibration is semi-Kan, $p^{\prime}: E^{\prime} \rightarrow B$ is a semi-Kan fibration by Proposition 4.4. Furthermore, since $\varepsilon_{n}(x)=\varepsilon_{n}(y)$ for any pair of $n$-simplices $x, y \in E_{n}$ such that $x \stackrel{(p)}{\sim} y$, it is easy to check that $p^{\prime}: E^{\prime} \rightarrow B$ is minimal. This completes the proof of Theorem 2.2.

Proof of Proposition 4.3. We must define a continuous section $\beta: E_{n} / \stackrel{(p)}{\sim} \rightarrow E_{n}$ of the quotient map $q: E_{n} \rightarrow E_{n} / \stackrel{(p)}{\sim}$ which satisfies the degeneracy condition $\beta\left(q\left(s_{k} e\right)\right)=s_{k} e$. We will need the following two lemmas. 
Lemma 4.5. Let $p: E \rightarrow B$ be a semi-Kan fibration. Then for each $n$ there exist continuous mappings

$$
\begin{gathered}
\varphi:\left\{(x, z) \in E_{n} \times E_{n} \mid \partial_{i} x=\partial_{i} z, p x=p z\right\} \rightarrow E_{n}, \\
\psi:\left\{(y, z) \in E_{n} \times E_{n} \mid \partial_{i} y=s_{0}^{n-1} \partial_{0}{ }^{n} z, p y=s_{0}{ }^{n} \partial_{0}{ }^{n} p z\right\} \rightarrow E_{n},
\end{gathered}
$$

which satisfy the following properties. (For brevity, we will write $\varphi_{z}$ and $\psi_{z}$ for $\varphi(\cdot, z)$ and $\psi(\cdot, z)$, respectively, where $z \in E_{n}$ is a fixed $n$-simplex.)

(i) $\partial_{i} \varphi_{z}(x)=s_{0}^{n-1} \partial_{0}^{n} z$ and $p \varphi_{z}(x)=s_{0}^{n} \partial_{0}^{n} p z$;

(ii) $\partial_{i} \psi_{z}(y)=\partial_{i} z$ and $p \psi_{z}(y)=p z$;

(iii) $x \stackrel{(p)}{\sim} x^{\prime} \Leftrightarrow \varphi_{z}(x) \stackrel{(p)}{\sim} \varphi_{z}\left(x^{\prime}\right)$;

(iv) $y \stackrel{(p)}{\sim} y^{\prime} \Leftrightarrow \psi_{z}(y) \stackrel{(p)}{\sim} \psi_{z}\left(y^{\prime}\right)$;

(v) $\psi_{z} \circ \varphi_{z}(x) \stackrel{(p)}{\sim} x$;

(vi) $\varphi_{z} \circ \psi_{z}(y) \stackrel{(p)}{\sim} y$;

(vii) $\varphi_{z}(z)=s_{0}^{n} \partial_{0}^{n} z$

(viii) $\psi_{z}\left(s_{0}^{n} \partial_{0}^{n} z\right)=z$.

Lemma 4.6. Let $p: E \rightarrow B$ be a semi-Kan fibration. Then for each $n$ there exist continuous mappings

$$
\begin{gathered}
\sigma:\left\{(x, a) \in E_{n} \times E_{1} \mid \partial_{i} x=s_{0}^{n-1} \partial_{1} a, p x=s_{0}^{n} \partial_{1} p a\right\} \rightarrow E_{n}, \\
\tau:\left\{(y, a) \in E_{n} \times E_{1} \mid \partial_{i} y=s_{0}^{n-1} \partial_{0} a, p y=s_{0}^{n} \partial_{0} p a\right\} \rightarrow E_{n},
\end{gathered}
$$

which satisfy the following properties. (For brevity, we write $\sigma_{a}$ and $\tau_{a}$ for $\sigma(\cdot, a)$ and $\tau(\cdot, a)$, respectively, where $a \in E_{1}$ is a fixed 1-simplex.)

(i) $\partial_{i} \sigma_{a}(x)=s_{0}^{n-1} \partial_{0} a$ and $p \sigma_{a}(x)=s_{0}^{n} \partial_{0} p a$;

(ii) $\partial_{i} \tau_{a}(y)=s_{0}^{n-1} \partial_{1} a$ and $p \tau_{a}(y)=s_{0}^{n} \partial_{1} p a$;

(iii) $x \stackrel{(p)}{\sim} x^{\prime} \Leftrightarrow \sigma_{a}(x) \stackrel{(p)}{\sim} \sigma_{a}\left(x^{\prime}\right)$;

(iv) $y \stackrel{(p)}{\sim} y^{\prime} \Leftrightarrow \tau_{a}(y) \stackrel{(p)}{\sim} \tau_{a}\left(y^{\prime}\right)$;

(v) $\tau_{a} \circ \sigma_{a}(x) \stackrel{(p)}{\sim} x$;

(vi) $\sigma_{a} \circ \tau_{a}(y) \stackrel{(p)}{\sim} y$;

(vii) $\sigma_{a}\left(s_{0}^{n} \partial_{1} a\right)=s_{0}^{n} \partial_{0} a$;

(viii) $\tau_{a}\left(s_{0}^{n} \partial_{0} a\right)=s_{0}^{n} \partial_{1} a$.

Before proving these two lemmas, we complete the proof of Proposition 4.3 by defining $\beta: E_{n} / \stackrel{(p)}{\sim} \rightarrow E_{n}$. Let $u \in E_{n} / \stackrel{(p)}{\sim}$, and choose a representative $e \in E_{n}$ of the $p$-homotopy equivalence class of $u$. Let

$$
z=c_{n}\left(p e ; \partial_{0} e, \ldots, \partial_{n} e\right) \in E_{n},
$$

where $c_{n}: E(n) \rightarrow E_{n}$ is a continuous section of $\rho_{n}: E_{n} \rightarrow E(n)$ satisfying the degeneracy condition $c_{n}\left(\rho_{n}\left(s_{k} e\right)\right)=s_{k} e$ for $e \in E_{n-1}$ and $0 \leq k \leq n-1$. Note that $z$ is independent of the choice of $e$. Let $w=c_{1}\left(* ; *, \partial_{0}^{n} z\right) \in F_{1}$, 
where $c_{1}: E(1) \rightarrow E_{1}$ is a continuous section of $\rho_{1}: E_{1} \rightarrow E(1)$ satisfying the degeneracy condition $c_{1}\left(\rho_{1}\left(s_{0} e\right)\right)=s_{0} e$ for $e \in E_{0}$. (Note that we must have $B_{0}=*$ in order for $w$ to be defined for every $u \in E_{n} / \stackrel{(p)}{\sim}$.) Define the continuous mapping $\beta: E_{n} / \stackrel{(p)}{\sim} \rightarrow E_{n}$ by

$$
\beta(u)=\psi_{z} \circ \tau_{w} \circ \alpha \circ \nu \circ \sigma_{w} \circ \varphi_{z}(e),
$$

where $\alpha: \pi_{n} F \rightarrow \widetilde{F}_{n}$ is a continuous section of the quotient map $\nu: \widetilde{F}_{n} \rightarrow$ $\pi_{n} F$ satisfying the degeneracy condition $\alpha(0)=*$. Then by parts (iii)-(vi) of Lemmas 4.5 and 4.6, $\beta$ is a well-defined map and $q(\beta(u))=u$. Furthermore, by parts (vii) and (viii) of these lemmas, $\beta$ satisfies the degeneracy condition $\beta\left(q\left(s_{k} e\right)\right)=s_{k} e$.

Proof of Lemma 4.5. We first define the mapping $\varphi$. For $n>0$, let $(x, z) \in$ $E_{n} \times E_{n}$ so that $\partial_{i} x=\partial_{i} z$ for all $i$, and so that $p x=p z$. We define $\varphi_{z}(x)$ by an inductive construction. For $0 \leq k \leq n$, let $z_{k}=s_{n-k}^{k} \partial_{0}^{k} z \in E_{n}$. Then $z_{0}=z, z_{n}=s_{0}^{n} \partial_{0}^{n} z$, and $z_{k}=\partial_{0} s_{n} z_{k-1}$. For $0 \leq k \leq n$ let $x_{k} \in E_{n}$ be defined inductively as follows. Let $x_{0}=x$. Assuming $x_{k}$ is defined so that $\partial_{i} x_{k}=\partial_{i} z_{k}$ and $p x_{k}=p z_{k}$, define

$$
x_{k+1}=\partial_{0} \lambda\left(s_{n} p z_{k} ;-, \partial_{1} s_{n} z_{k}, \ldots, \partial_{n-1} s_{n} z_{k}, x_{k}, z_{k}\right) \text {. }
$$

Then $\partial_{i} x_{k+1}=\partial_{i} z_{k+1}$ and $p x_{k+1}=p z_{k+1}$.

Define $\varphi_{z}(x)=x_{n}$. Then $\partial_{i} \varphi_{z}(x)=\partial_{i} x_{n}=s_{0}^{n-1} \partial_{0}^{n} z$ and $p \varphi_{z}(x)=p x_{n}=$ $s_{0}^{n} \partial_{0}^{n} p z$. This proves part (i) of Lemma 4.5.

We now define the second mapping, $\psi$. For $n>0$, let $(y, z) \in E_{n} \times E_{n}$ so that $\partial_{i} y=s_{0}^{n-1} \partial_{0}^{n} z$ for all $i$, and so that $p y=s_{0}^{n} \partial_{0}^{n} p z$. We define $\psi_{z}(y)$ by an inductive construction.

For $0 \leq k \leq n$ define $z_{k}=s_{n-k}^{k} \partial_{0}^{k} z \in E_{n}$ as above. For $0 \leq k \leq n$ define $y_{k} \in E_{n}$ inductively from $k=n$ to $k=0$ as follows. Let $y_{n}=y$. Assuming $y_{k+1}$ is defined so that $\partial_{i} y_{k+1}=\partial_{i} z_{k+1}$ and $p y_{k+1}=p z_{k+1}$, define

$$
y_{k}=\partial_{n} \lambda\left(s_{n} p z_{k} ; y_{k+1}, \partial_{1} s_{n} z_{k}, \ldots, \partial_{n-1} s_{n} z_{k},-, z_{k}\right) \text {. }
$$

Then $\partial_{i} y_{k}=\partial_{i} z_{k}$ and $p y_{k}=p z_{k}$.

Define $\psi_{z}(y)=y_{0}$. Then $\partial_{i} \psi_{z}(y)=\partial_{i} y_{0}=\partial_{i} z$ and $p \psi_{z}(y)=p y_{0}=p z$. This proves part (ii) of Lemma 4.5.

Parts (iv) and (v) of Lemma 4.5 follow easily from (iii) and (vi), while (vii) and (viii) follow from straightforward inductive arguments. Parts (iii) and (vi) are also inductive arguments and require the following two lemmas. We leave the details to the reader.

Lemma 4.7. Let $u=\lambda\left(b ;-, e_{1}, \ldots, e_{n+1}\right)$ and

$$
u^{\prime}=\lambda\left(b ;-, e_{1}, \ldots, e_{n-1}, e_{n}^{\prime}, e_{n+1}\right) \text {. }
$$

Then $e_{n} \stackrel{(p)}{\sim} e_{n}^{\prime}$ if and only if $\partial_{0} u \stackrel{(p)}{\sim} \partial_{0} u^{\prime}$. 
Proof. Let $z: e_{n} \stackrel{(p)}{\sim} e_{n}^{\prime}$, and define

$$
\theta=\partial_{0} \lambda\left(s_{n+1} b ;-, s_{n} e_{1}, \ldots, s_{n} e_{n-1}, z, u, u^{\prime}\right) .
$$

Then $\theta: \partial_{0} u \stackrel{(p)}{\sim} \partial_{0} u^{\prime}$. Conversely, let $\theta: \partial_{0} u \stackrel{(p)}{\sim} \partial_{0} u^{\prime}$, and define

$$
z=\partial_{n} \lambda\left(s_{n+1} b ; \theta, s_{n} e_{1}, \ldots, s_{n} e_{n-1},-, u, u^{\prime}\right) .
$$

Then $z: e_{n} \stackrel{(p)}{\sim} e_{n}^{\prime}$.

Lemma 4.8. Let $u=\lambda\left(b ; e_{0}, \ldots, e_{n-1},-, e_{n+1}\right)$ and

$$
v=\lambda\left(b ;-, e_{1}, \ldots, e_{n-1}, \partial_{n} u, e_{n+1}\right) \text {. }
$$

Then $e_{0} \stackrel{(p)}{\sim} \partial_{0} v$.

Proof. Define $\theta=\partial_{0} \lambda\left(b ;-, s_{n} e_{1}, \ldots, s_{n} e_{n-1}, s_{n} \partial_{n} u, u, v\right)$. Then $\theta: e_{0} \stackrel{(p)}{\sim}$ $\partial_{0} v$.

Proof of Lemma 4.6. We first construct the mapping $\sigma$. For $n>0$, let $(x, a) \in$ $E_{n} \times E_{1}$ so that $\partial_{i} x=s_{0}^{n-1} \partial_{1} a$ for all $i$, and so that $p x=s_{0}^{n} \partial_{1} p a$. We define $\sigma_{a}(x)$ by an inductive construction. For $1 \leq k \leq n$, define

$$
a_{k}=s_{n-1} \cdots \widehat{s_{n-k}} \cdots s_{0} a \in E_{n} \text {. }
$$

Then $a_{k+1}=\partial_{0} s_{n} a_{k}, \partial_{0} a_{k}=\partial_{n-1} a_{k+1}=\partial_{n} a_{k+1}, \partial_{0} a_{n}=s_{0}^{n-1} \partial_{0} a, \partial_{n} a_{1}=$ $s_{0}^{n-1} \partial_{1} a$, and for $0 \leq i<n, \partial_{i} a_{1}=s_{0}^{n-2} a$.

For $0 \leq k \leq n+1$ define $x_{k} \in E_{n}$ inductively as follows. Let $x_{0}=x$ and

$$
x_{1}=\partial_{0} \lambda\left(s_{0}^{n} p a ;-, a_{1}, \ldots, a_{1}, x_{0}\right) \text {. }
$$

Then $\partial_{i} x_{1}=\partial_{i} a_{1}$ for all $i$, and $p x_{1}=p a_{1}$. Assuming $x_{k}$ is defined so that $\partial_{i} x_{k}=\partial_{i} a_{k}$ for all $i$ and $p x_{k}=p a_{k}$, define

$$
x_{k+1}=\partial_{0} \lambda\left(s_{n} p a_{k} ;-, \partial_{1} s_{n} a_{k}, \ldots, \partial_{n-1} s_{n} a_{k}, a_{k}, x_{k}\right) .
$$

Then for $k<n, \partial_{i} x_{k+1}=\partial_{i} a_{k+1}$ for all $i$ and $p x_{k+1}=p a_{k+1}$.

Define $\sigma_{a}(x)=x_{n+1}$. Then $\partial_{i} \sigma_{a}(x)=\partial_{i} x_{n+1}=s_{0}^{n-1} \partial_{0} a$ for all $i$, and $p \sigma_{a}(x)=p x_{n+1}=s_{0}^{n} \partial_{0} p a$. This proves part (i) of Lemma 4.6.

We now define the second mapping, $\tau$. For $n>0$, let $(y, a) \in E_{n} \times E_{1}$ so that $\partial_{i} y=s_{0}^{n-1} \partial_{0} a$ for all $i$, and so that $p y=s_{0}^{n} \partial_{0} p a$. We define $\tau_{a}(y)$ by an inductive construction.

For $1 \leq k \leq n$ define $a_{k} \in E_{n}$ as above. For $0 \leq k \leq n+1$ define $y_{k} \in E_{n}$ inductively from $k=n+1$ to $k=0$ as follows. Let $y_{n+1}=y$. Let $k>0$ and assume $y_{k+1}$ is defined so that $\partial_{i} y_{k+1}=\partial_{i} a_{k+1}$ for all $i$ and $p y_{k+1}=p a_{k+1}$. Define

$$
y_{k}=\partial_{n+1} \lambda\left(s_{n} p a_{k} ; y_{k+1}, \partial_{1} s_{n} a_{k}, \ldots, \partial_{n-1} s_{n} a_{k}, a_{k},-\right) .
$$

Then $\partial_{i} y_{k}=\partial_{i} a_{k}$ for all $i$ and $p y_{k}=p a_{k}$. Finally, define

$$
y_{0}=\partial_{n+1} \lambda\left(s_{0}^{n} p a ; y_{1}, a_{1}, \ldots, a_{1},-\right) .
$$


Define $\tau_{a}(y)=y_{0}$. Then $\partial_{i} \tau_{a}(y)=\partial_{i} y_{0}=s_{0}^{n-1} \partial_{1} a$ for all $i$, and $p \tau_{a}(y)=$ $p y_{0}=s_{0}^{n} \partial_{1} p a$. This proves part (ii) of Lemma 4.6.

The proofs of parts (iii)-(viii) of Lemma 4.6 are analogous to those of Lemma 4.5 , and we leave them to the reader.

\section{Proof of Theorem 1.4}

Let $f: X \rightarrow Y$ be a strong equivalence of minimal, semi-Kan spaces. Thus, the induced homomorphism $f_{*}: \pi_{n} X \rightarrow \pi_{n} Y$ is a homeomorphism. We must show that $f$ is a simplicial homeomorphism.

Lemma 5.1. Let $f: X \rightarrow Y$ be a continuous simplicial map of minimal, semiKan spaces. Then there exist continuous mappings

$$
\begin{gathered}
\varphi_{X}:\left\{(x, z) \in X_{n} \times X_{n} \mid \partial_{i} x=\partial_{i} z\right\} \rightarrow \pi_{n} X, \\
\varphi_{Y}:\left\{(y, z) \in Y_{n} \times Y_{n} \mid \partial_{i} y=\partial_{i} z\right\} \rightarrow \pi_{n} Y, \\
\psi_{X}: \pi_{n} X \times X_{n} \rightarrow X_{n}, \\
\psi_{Y}: \pi_{n} Y \times Y_{n} \rightarrow Y_{n},
\end{gathered}
$$

which satisfy the following conditions:

(i) $\psi_{x}\left(\varphi_{x}(x, z), z\right)=x$ for $x, z \in X_{n}$ with $\partial_{i} x=\partial_{i} z$;

(ii) $\psi_{Y}\left(\varphi_{Y}(y, z), z\right)=y$ for $y, z \in Y_{n}$ with $\partial_{i} y=\partial_{i} z$;

(iii) $\varphi_{Y}(f(x), f(z))=f_{*}\left(\varphi_{X}(x, z)\right)$ for $x, z \in X_{n}$ with $\partial_{i} x=\partial_{i} z$;

(iv) $\psi_{Y}\left(f_{*}(\alpha), f(z)\right)=f\left(\psi_{X}(\alpha, z)\right)$ for $\alpha \in \pi_{n} X$ and $z \in X_{n}$.

Proof. Let $\varphi_{X}$ and $\psi_{x}$ be the mappings $\varphi$ and $\psi$ of Lemma 4.5 applied to the trivial semi-Kan fibration $X \rightarrow *$. Define $\varphi_{Y}$ and $\psi_{Y}$ similarly. Parts (i) and (ii) of Lemma 5.1 then follow immediately from Lemma 4.5(v), and parts (iii) and (iv) follow from a straightforward inductive argument.

We now prove by induction that the strong equivalence $f: X \rightarrow Y$ is a simplicial homeomorphism. The case $n=0$ is trivial since $X_{0}$ and $Y_{0}$ consist of a single basepoint. Assuming $f: X_{n-1} \rightarrow Y_{n-1}$ is a homeomorphism, define a continuous mapping $g=g_{n}: Y_{n} \rightarrow X_{n}$ by

$$
g(y)=\psi_{X}\left(f_{*}^{-1} \circ \varphi_{Y}(y, f \circ \eta(y)), \eta(y)\right),
$$

where $\eta(y)=\lambda_{X}\left(-, f^{-1}\left(\partial_{1} y\right), \ldots, f^{-1}\left(\partial_{n} y\right)\right)$. We claim that $g$ is the inverse of $f: X_{n} \rightarrow Y_{n}{ }^{x}$. We have

$$
\begin{aligned}
f \circ g(y) & =f \circ \psi_{X}\left(f_{*}^{-1} \circ \varphi_{Y}(y, f \circ \eta(y)), \eta(y)\right) \\
& =\psi_{Y}\left(f_{*} \circ f_{*}^{-1} \circ \varphi_{Y}(y, f \circ \eta(y)), f \circ \eta(y)\right) \quad \text { by Lemma 5.1(iv) } \\
& =\psi_{Y}\left(\varphi_{Y}(y, f \circ \eta(y)), f \circ \eta(y)\right) \\
& =y \quad \text { by Lemma } 5.1(\mathrm{ii}) .
\end{aligned}
$$


Similarly, we have $g \circ f(x)=x$, whence $f: X_{n} \rightarrow Y_{n}$ is a homeomorphism.

\section{Proof of Theorem 2.7}

We need to prove that a mapping $p: E \rightarrow B$, where $B_{0}=*$, is a minimal, semi-Kan fibration if and only if it is a twisted product with minimal, semi-Kan fiber. If $p: E \rightarrow B$ is a twisted product with minimal, semi-Kan fiber, then an easy adaptation of [3, Lemma $6.7 ; 6$, Proposition 18.4 (ii)] shows that it is a minimal, semi-Kan fibration.

Conversely, suppose $p: E \rightarrow B$ is a minimal, semi-Kan fibration. We will define fiber bundles and prove that any fiber bundle with a normalized, regular atlas is a twisted product. We will then prove that $p: E \rightarrow B$ is a fiber bundle with normalized, regular atlas, hence a twisted product.

For $b \in B_{n}$, let $\bar{b}: \Delta[n] \rightarrow B$ denote the simplicial map determined by $b$, and define $E^{b}$ to be the pullback of $p: E \rightarrow B$ by the map $\bar{b}: \Delta[n] \rightarrow B$.

Definition 6.1. A fiber bundle with fiber $F$ is a continuous simplicial surjective map $p: E \rightarrow B$ of simplicial spaces such that for every $n \geq 0$ and $b \in B_{n}$ there is a simplicial homeomorphism $\alpha(b): F \times \Delta[n] \rightarrow E^{b}$ such that the following diagram commutes:

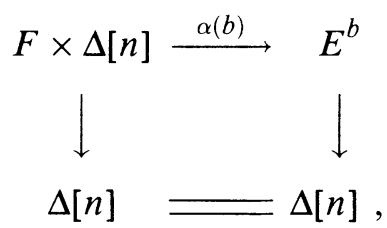

where the vertical mappings are the natural projections. (Cf. [6, §19].) We also require the following continuity conditions. For every $n$ the mapping $\alpha: B_{n} \rightarrow \mathfrak{F}(F, E)_{n}$, determined by $\left\{\alpha(b) \mid b \in B_{n}\right\}$, must be continuous; and the mappings

$$
\beta=\beta_{n, q}:\left\{(b, e, u) \in B_{n} \times E_{q} \times \Delta[n]_{q} \mid(e, u) \in E_{q}^{b}\right\} \rightarrow F_{q} \times \Delta[n]_{q},
$$

defined by $\beta(b, e, u)=\alpha(b)^{-1}(e, u)$, must also be continuous.

The mapping $\alpha: B \rightarrow \mathfrak{F}(F, E)$, called the atlas of the fiber bundle, need not be a simplicial map. If $\alpha \circ s_{k}=s_{k} \circ \alpha$ for all $k$, then the atlas $\alpha$ is said to be normalized, and if $\alpha \circ \partial_{k}=\partial_{k} \circ \alpha$ for $k>0$, then $\alpha$ is said to be regular.

Proposition 6.2. Let $p: E \rightarrow B$ be a fiber bundle with fiber $F$ and normalized, regular atlas $\alpha: B \rightarrow \mathfrak{F}(F, E)$. Then $p: E \rightarrow B$ is a twisted product.

Proof. For $n>0$ we will construct a twisting function $\tau=\tau_{n}: B_{n} \rightarrow \mathfrak{A}(F)_{n-1}$ and a simplicial homeomorphism $\xi: B \times{ }_{\tau} F \rightarrow E$ such that $p \circ \xi(b, x)=b$ for $(b, x) \in\left(B \times{ }_{\tau} F\right)_{q}, q \geq 0$. The construction of $\tau$ is adapted from [6, $\left.\S 19\right]$. Let $b \in B_{n}$, and let $\delta_{0}: \Delta[n-1] \hookrightarrow \Delta[n]$ be the natural simplicial inclusion 
map defined by $\delta_{0}\left(\Delta_{n-1}\right)=\partial_{0} \Delta_{n}$. Then the following diagram commutes:

$$
\begin{aligned}
& F \times \Delta[n] \stackrel{\alpha(b)}{\longrightarrow} E^{b} \\
& \operatorname{id}_{F} \times \delta_{0} \uparrow \quad \uparrow \operatorname{id}_{E} \times \delta_{0} \\
& F \times \Delta[n-1] \underset{\partial_{0} \alpha(b)}{\longrightarrow} E^{\partial_{0} b}
\end{aligned}
$$

Since the mapping $\alpha(b)$ is a simplicial homeomorphism, so are the mappings $\partial_{0} \alpha(b)$ and $\alpha\left(\partial_{0} b\right)$. Thus, we can define a simplicial homeomorphism $\tau(b)$ : $F \times \Delta[n-1] \rightarrow F \times \Delta[n-1]$ by

$$
\tau(b)=\alpha\left(\partial_{0} b\right)^{-1} \circ \partial_{0} \alpha(b) \text {. }
$$

We regard $\tau(b)$ as an element of $\mathfrak{A}(F)_{n-1}$ by composing it with the natural projection of $F \times \Delta[n-1]$ onto $F$. Since we are working in the category of compactly generated spaces, the mapping $\tau: B_{n} \rightarrow \mathfrak{A}(F)_{n-1}$ is continuous.

To show that $\tau$ is a twisting map, it suffices to construct a simplicial homeomorphism $\xi: B \times{ }_{\tau} F \rightarrow E$. Define $\xi$ by $\xi(b, x)=\alpha(b)\left(x, \Delta_{n}\right)$ for $(b, x) \in$ $B_{n} \times F_{n}$, and define a mapping $\eta: E \rightarrow B \times{ }_{\tau} F$ by $\eta(e)=\left(p e, \alpha(p e)^{-1}\left(e, \Delta_{n}\right)\right)$, $e \in E_{n}$. It is easy to check that $\xi \circ \eta=\mathrm{id}_{E}, \eta \circ \xi=\operatorname{id}_{B \times_{F} F}$, and $p \circ \xi(b, x)=b$. This completes the proof of Proposition 6.2.

Let $\sigma_{k}: \Delta[n] \rightarrow \Delta[n-1]$ and $\delta_{k}: \Delta[n] \rightarrow \Delta[n+1]$ be the canonical simplicial maps defined by $\sigma_{k}\left(\Delta_{n}\right)=s_{k} \Delta_{n-1}$ and $\delta_{k}\left(\Delta_{n}\right)=\partial_{k} \Delta_{n+1}$. We will need the following lemma.

Lemma 6.3. Let $p: E \rightarrow B$ be a minimal, semi-Kan fibration with fiber $F$, and assume $B_{0}=*$. Then for $n, q \geq 0$ there exist continuous mappings

$$
\begin{gathered}
f=f_{n, q}: B_{n} \times F_{q} \times \Delta[n]_{q} \rightarrow E_{q}, \\
g=g_{n, q}:\left\{(b, e, u) \in B_{n} \times E_{q} \times \Delta[n]_{q} \mid(e, u) \in E_{q}^{b}\right\} \rightarrow F_{q},
\end{gathered}
$$

which satisfy the following conditions:

(i) $(f(b, x, u), u) \in E_{q}^{b} \quad$ for $(b, x, u) \in B_{n} \times F_{q} \times \Delta[n]_{q}$;

(ii) $\partial_{i} f(b, x, u)=f\left(b, \partial_{i} x, \partial_{i} u\right)$ and $s_{i} f(b, x, u)=f\left(b, s_{i} x, s_{i} u\right)$ for all $i$

(iii) $f(b, g(b, e, u), u)=e$;

(iv) $g(b, f(b, x, u), u)=x$;

(v) $f\left(s_{k} b, x, u\right)=f\left(b, x, \sigma_{k} u\right)$ for all $k$, where $(b, x, u) \in B_{n-1} \times F_{q} \times$ $\Delta[n]_{q}$

(vi) $f\left(\partial_{k} b, x, u\right)=f\left(b, x, \delta_{k} u\right)$ for $k>0$, where $(b, x, u) \in B_{n+1} \times$ $F_{q} \times \Delta[n]_{q}$.

Before proving this lemma, we complete the proof of Theorem 2.7. We must define an atlas $\alpha: B \rightarrow \mathfrak{F}(F, E)$ for the minimal, semi-Kan fibration $p: E \rightarrow$ $B$. Let $b \in B_{n}$, and define a continuous mapping $\alpha(b): F \times \Delta[n] \rightarrow E^{b}$ by 
$\alpha(b)(x, u)=(f(b, x, u), u)$. Then $\alpha(b)$ is a simplicial map by Lemma 6.3(ii). Define a continuous mapping

$$
\beta:\left\{(b, e, u) \in B_{n} \times E_{q} \times \Delta[n]_{q} \mid(e, u) \in E_{q}^{b}\right\} \rightarrow F_{q} \times \Delta[n]_{q}
$$

by $\beta(b, e, u)=(g(b, e, u), u)$. Then parts (iii) and (iv) of Lemma 6.3 imply that $\alpha(b): F \times \Delta[n] \rightarrow E^{b}$ is a simplicial homeomorphism with inverse defined by $\alpha(b)^{-1}(e, u)=\beta(b, e, u)$. Parts (v) and (vi) of this lemma imply that $\alpha$ is normalized and regular. Thus, by Definition 6.1, $p: E \rightarrow B$ is a fiber bundle with normalized, regular atlas $\alpha: B \rightarrow \mathfrak{F}(F, E)$.

Proof of Proposition 6.3. Before defining the mapping $f$ of Lemma 6.3, we need to construct mappings $h_{i}, \widetilde{h}_{i}$, and $f_{i}$. For $n \geq 0$, the simplicial set $\Delta[n]$ is contractible [6, Lemma 11.10], and there is a contracting homotopy given by the homotopy maps $h_{i}: \Delta[n]_{q} \rightarrow \Delta[n]_{q+1}$ defined by $h_{0}\left(\Delta_{n}\right)=h_{1}\left(\Delta_{n}\right)=s_{0} \Delta_{n}$ and

$$
h_{i}\left(\Delta_{n}\right)=s_{i-1} \cdots s_{0} \partial_{1} \cdots \partial_{i-1} \Delta_{n} \text { for } 1<i \leq n .
$$

Note that $\partial_{0} h_{0} u=u$ and $\partial_{q+1} h_{q} u=\overline{0}$ for $u \in \Delta[n]_{q}$. For $b \in B_{n}$, let $\bar{b}: \Delta[n] \rightarrow B$ be the simplicial map determined by $b$. For $q \geq 0$ and $0 \leq i \leq q$, define continuous mappings $\widetilde{h}_{i}: B_{n} \times \Delta[n]_{q} \rightarrow B_{q+1}$ by $\widetilde{h}_{i}(b, u)=\bar{b} \circ h_{i}(u)$. Note that $\partial_{q+1} \widetilde{h}_{q}(b, u)=*$.

We will use the $\widetilde{h}_{i}$ 's and double induction on $q$ and $i$ to define continuous mappings $f_{i}: B_{n} \times F_{q} \times \Delta[n]_{q} \rightarrow E_{q+1}$ for $q \geq 0$ and $0 \leq i \leq q$. For $q=0$, define $f_{0}: B_{n} \times F_{0} \times \Delta[n]_{0} \rightarrow E_{1}$ by

$$
f_{0}(b, x, u)=\lambda\left(\widetilde{h}_{0}(b, u) ;-, x\right) \text {. }
$$

For the first inductive step, let $q>0$ and assume $f_{i}: B_{n} \times F_{q-1} \times \Delta[n]_{q-1} \rightarrow E_{q}$ is defined for all $i$. Define $f_{q}: B_{n} \times F_{q} \times \Delta[n]_{q} \rightarrow E_{q+1}$ by

$$
\begin{array}{r}
f_{q}(b, x, u)=\lambda\left(\widetilde{h}_{q}(b, u) ; f_{q-1}\left(b, \partial_{0} x, \partial_{0} u\right), \ldots,\right. \\
\left.f_{q-1}\left(b, \partial_{q-1} x, \partial_{q-1} u\right),-, x\right),
\end{array}
$$

where $(b, x, u) \in B_{n} \times F_{q} \times \Delta[n]_{q}$. For the second inductive step, let $0<k<q$ and assume $f_{k+1}: B_{n} \times F_{q} \times \Delta[n]_{q} \rightarrow E_{q+1}$ is defined. Define $f_{k}: B_{n} \times F_{q} \times$ $\Delta[n]_{q} \rightarrow E_{q+1}$ by

$$
\begin{array}{r}
f_{k}(b, x, u)=\lambda\left(\tilde{h}_{k}(b, u) ; f_{k-1}\left(b, \partial_{0} x, \partial_{0} u\right), \ldots, f_{k-1}\left(b, \partial_{k-1} x, \partial_{k-1} u\right),\right. \\
-, \partial_{k+1} f_{k+1}(b, x, u), f_{k}\left(b, \partial_{k+1} x, \partial_{k+1} u\right), \ldots, \\
\left.f_{k}\left(b, \partial_{q} x, \partial_{q} u\right)\right) .
\end{array}
$$

Finally, define $f_{0}: B_{n} \times F_{q} \times \Delta[n]_{q} \rightarrow E_{q+1}$ by

$$
\begin{aligned}
f_{0}(b, x, u)=\lambda\left(\tilde{h}_{0}(b, u)\right. & ;-, \partial_{1} f_{1}(b, x, u), \\
& \left.f_{0}\left(b, \partial_{1} x, \partial_{1} u\right), \ldots, f_{0}\left(b, \partial_{q} x, \partial_{q} u\right)\right) .
\end{aligned}
$$


Define $f: B_{n} \times F_{q} \times \Delta[n]_{q} \rightarrow E_{q}$ by $f(b, x, u)=\partial_{0} f_{0}(b, x, u)$. Then

$$
p f(b, x, u)=p \partial_{0} f_{0}(b, x, u)=\partial_{0} \widetilde{h}_{0}(b, u)=\bar{b}\left(\partial_{0} h_{0}(u)\right)=\bar{b}(u) .
$$

This proves part (i) of Lemma 6.3.

The proof that $\partial_{i} f(b, x, u)=f\left(b, \partial_{i} x, \partial_{i} u\right)$ is straightforward. To prove that $s_{i} f(b, x, u)=f\left(b, s_{i} x, s_{i} u\right)$, argue by induction, using the degeneracy condition for the semi-Kan maps $\lambda$ and the fact that the $h_{i}$ 's are homotopy maps, to show that

$$
f_{i}\left(b, s_{j} x, s_{j} u\right)= \begin{cases}s_{j+1} f_{i}(b, x, u) & \text { if } i \leq j \\ s_{j} f_{i-1}(b, x, u) & \text { if } i>j .\end{cases}
$$

This proves part (ii).

We now define the continuous mapping $g$ of Lemma 6.3. To do so, we must construct continuous mappings

$$
g_{i}:\left\{(b, e, u) \in B_{n} \times E_{q} \times \Delta[n]_{q} \mid(e, u) \in E_{q}^{b}\right\} \rightarrow E_{q+1}
$$

for $q \geq 0$ and $0 \leq i \leq q$. For brevity, let us define

$$
T_{n, q}=\left\{(b, e, u) \in B_{n} \times E_{q} \times \Delta[n]_{q} \mid(e, u) \in E_{q}^{b}\right\} .
$$

Define $g_{0}: T_{n, 0} \rightarrow E_{1}$ by $g_{0}(b, e, u)=\lambda\left(\widetilde{h}_{0}(b, u) ; e,-\right)$. For the first inductive step, let $q>0$ and assume $g_{i}: T_{n, q-1} \rightarrow E_{q}$ is defined for all $i$. Let $(b, e, u) \in T_{n, q}$. For ease of notation, define

$$
c_{i}=\left(b, \partial_{q} g_{q-1}\left(b, \partial_{i} e, \partial_{i} u\right), \partial_{i} u\right) \in B_{n} \times F_{q-1} \times \Delta[n]_{q-1},
$$

and define $g_{0}: T_{n, q} \rightarrow E_{q+1}$ by

$$
g_{0}(b, e, u)=\lambda\left(\widetilde{h}_{0}(b, u) ; e,-, f_{0}\left(c_{1}\right), \ldots, f_{0}\left(c_{q}\right)\right) .
$$

For the second inductive step, let $0<k<q$ and assume $g_{k-1}: T_{n, q} \rightarrow E_{q+1}$ is defined. Define $g_{k}: T_{n, q} \rightarrow E_{q+1}$ by

$$
\begin{array}{r}
g_{k}(b, e, u)=\lambda\left(\widetilde{h}_{k}(b, u) ; f_{k-1}\left(c_{0}\right), \ldots, f_{k-1}\left(c_{k-1}\right), \partial_{k} g_{k-1}(b, e, u),\right. \\
\left.-, f_{k}\left(c_{k+1}\right), \ldots, f_{k}\left(c_{q}\right)\right) .
\end{array}
$$

Finally, define $g_{q}: T_{n, q} \rightarrow E_{q+1}$ by

$$
g_{q}(b, e, u)=\lambda\left(\widetilde{h}_{q}(u) ; f_{q-1}\left(c_{0}\right), \ldots, f_{q-1}\left(c_{q-1}\right), \partial_{q} g_{q-1}(b, e, u),-\right),
$$

and define $g: T_{n, q} \rightarrow F_{q}$ by $g(b, e, u)=\partial_{q+1} g_{q}(b, e, u)$.

Parts (iii)-(vi) of Lemma 6.3 follow from straightforward but tedious arguments using double induction and the fact that $p: E \rightarrow B$ is minimal. We only remark that to prove (v) one needs the following lemma. (An analogous lemma is needed for part (vi).) 
Lemma 6.4. $\quad \tilde{h}_{i}\left(s_{k} b, u\right)=\widetilde{h}_{i}\left(b, \sigma_{k} u\right)$ for all $k$.

Proof. It suffices to show that the square in the following diagram commutes:

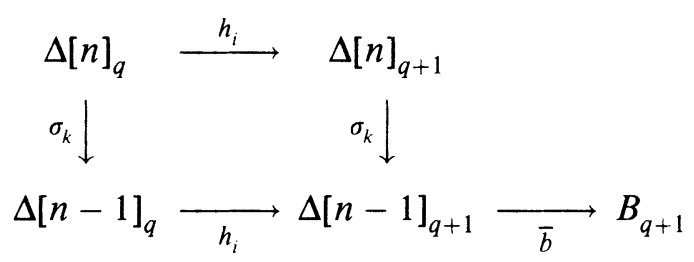

\section{Proof of Theorem 2.8}

Let $p: E=B \times{ }_{\tau} F \rightarrow B$ be a twisted product with minimal, semi-Kan fiber $F$ such that $B_{0}=*$. We need to define a sequence

$$
\cdots \rightarrow E^{(n)} \rightarrow E^{(n-1)} \rightarrow \cdots \rightarrow E^{(1)} \rightarrow E^{(0)}=B
$$

of twisted products such that the fiber of $E^{(n)} \rightarrow E^{(n-1)}$ is $K\left(\pi_{n} F, n\right)$, and such that $E_{q}=E_{q}^{(n)}$ for $q \leq n$. For $q, n \geq 0$, let $\stackrel{n}{\sim}$ be the equivalence relation on the $q$-simplices of $E$ defined by [6, Definition 8.8]. Let $E^{(n)}$ be the simplicial space whose space of $q$-simplices, $E_{q}^{(n)}$, is the quotient space $E_{q} / \stackrel{n}{\sim}$, with face and degeneracy maps induced from those of $E$. By [6, Definition 8.8], $E_{q}=E_{q}^{(n)}$ for $q \leq n$, and $E^{(0)}$ is simplicially homeomorphic to $B$ since $E_{0}=*$. For $0 \leq m \leq n \leq \infty$, let $p_{m}^{n}: E^{(n)} \rightarrow E^{(m)}$ be the natural projection map, and define $p^{n}=p_{0}^{n}: E^{(n)} \rightarrow B$.

Lemma 7.1. $p^{n}: E^{(n)} \rightarrow B$ is a semi-Kan fibration.

Using this lemma it is straightforward to show that $p_{m}^{n}: E^{(n)} \rightarrow E^{(m)}$ is a semi-Kan fibration, and by [6, Lemma 12.1] it is minimal. In particular, $p_{n-1}^{n}: E^{(n)} \rightarrow E^{(n-1)}$ is a minimal, semi-Kan fibration, hence a twisted product, whose fiber $Z$ is minimal and semi-Kan by Theorem 2.7. Since $\pi_{i} Z=0$ for $i \neq n$ and $\pi_{n} Z=\pi_{n} F$, we have $Z=K\left(\pi_{n} F, n\right)$ by Corollary 1.9. This completes the proof of Theorem 2.8 .

Proof of Lemma 7.1. Let $\lambda=\lambda_{q+1, k}: E(q+1, k) \rightarrow E_{q+1}$ denote the semi-Kan maps of $p: E \rightarrow B$. We will define semi-Kan maps

$$
\bar{\lambda}=\bar{\lambda}_{q+1, k}: E^{(n)}(q+1, k) \rightarrow E_{q+1}^{(n)}
$$

for $p^{n}: E^{(n)} \rightarrow B$ by an inductive construction.

Let $q$ and $n$ be positive integers. If $q \leq n$, then $E_{q}^{(n)}=E_{q}$, so $\bar{\lambda}_{q+1, k}$ is defined by $\lambda_{q+1, k}$ when $q \leq n$.

Assume $q>n$, and define $l=q-n$. For $x \in E_{q}$, let $[x] \in E_{q}^{(n)}$ denote the equivalence class of $x$. Let

$$
\left(b ;\left[x_{0}\right], \ldots,\left[x_{k-1}\right],-,\left[x_{k+1}\right], \ldots,\left[x_{q+1}\right]\right) \in E^{(n)}(q+1, k) .
$$


For each integer $j$ such that $1 \leq j \leq l$, let $i_{j}$ be an integer satisfying the following conditions:

$$
0 \leq i_{j} \leq n+j+1 \text { and } i_{j} \neq k .
$$

Since $\partial_{i} \partial_{i_{1}} \cdots \partial_{i_{l-1}}\left[x_{i_{l}}\right]=\partial_{i} \partial_{i-1} \cdots \partial_{i_{l-1}} x_{i_{l}} \in E_{n}$, we can define

$$
\begin{aligned}
y_{\left(i_{1}, \ldots, i_{l}\right)}=\lambda\left(\partial_{i_{1}} \cdots \partial_{i_{l}} b ;-, \partial_{1} \partial_{i_{1}} \cdots \partial_{i_{l-1}}\left[x_{i_{l}}\right], \ldots,\right. & , \\
& \left.\partial_{n+1} \partial_{i_{1}} \cdots \partial_{i_{l-1}}\left[x_{i_{l}}\right]\right) \in E_{n+1}
\end{aligned}
$$

for every possible $l$-tuple of integers $\left(i_{1}, \ldots, i_{l}\right)$ with the $i_{j}$ 's defined in (7.2) above. Since $p: E \rightarrow B$ is minimal, $\partial_{0} y_{\left(i_{1}, \ldots, i_{l}\right)}=\partial_{0} \partial_{i_{1}} \cdots \partial_{i_{l-1}}\left[x_{i_{l}}\right]$. Thus, $\left[y_{\left(i_{1}, \ldots, i_{l}\right)}\right]=\partial_{i_{1}} \cdots \partial_{i_{l-1}}\left[x_{i_{l}}\right] \in E_{n+1}^{(n)}$. For $i<j$ we have $\partial_{i} y_{\left(j, i_{2}, \ldots, i_{l}\right)}=$ $\partial_{j-1} y_{\left(i, i_{2}, \ldots, i_{l}\right)}$, and if $s$ is an integer such that $1 \leq s<l$ and $i_{s}<i_{s+1}$, then

$$
y_{\left(i_{1}, \ldots, i_{l}\right)}=y_{\left(i_{1}, \ldots, i_{s-1}, i_{s+1}-1, i_{s}, i_{s+2}, \ldots, i_{l}\right)} .
$$

For each integer $r$ such that $1 \leq r \leq l$ and each $(l-r+1)$-tuple $\left(i_{r}, \ldots, i_{l}\right)$ of integers $i_{j}$ satisfying (7.2), there is an $(n+r)$-simplex $y_{\left(i_{r}, \ldots, i_{l}\right)} \in E_{n+r}$ defined inductively by

$$
\begin{aligned}
y_{\left(i_{r}, \ldots, i_{l}\right)}=\lambda\left(\partial_{i_{r}} \cdots \partial_{i_{l}} b ; y_{\left(0, i_{r}, \ldots, i_{l}\right)}, \ldots, y_{\left(k-1, i_{r}, \ldots, i_{l}\right)},-,\right. & \\
& \left.y_{\left(k+1, i_{r}, \ldots, i_{l}\right)}, \ldots, y_{\left(n+r, i_{r}, \ldots, i_{l}\right)}\right)
\end{aligned}
$$

which satisfies

$$
\begin{gathered}
{\left[y_{\left(i_{r}, \ldots, i_{l}\right)}\right]=\partial_{i_{r}} \cdots \partial_{i_{l-1}}\left[x_{i_{l}}\right]} \\
p\left(y_{\left(i_{r}, \ldots, i_{l}\right)}\right)=\partial_{i_{r}} \cdots \partial_{i_{l}} b, \\
\partial_{i} y_{\left(j, i_{r+1}, \ldots, i_{l}\right)}=\partial_{j-1} y_{\left(i, i_{r+1}, \ldots, i_{l}\right)} \quad \text { for } i<j, \\
y_{\left(i_{r}, \ldots, i_{l}\right)}=y_{\left(i_{r}, \ldots, i_{s-1}, i_{s+1}-1, i_{s}, i_{s+2}, \ldots, i_{l}\right)} \quad \text { for } r \leq s<l \text { with } i_{s}<i_{s+1} .
\end{gathered}
$$

When $r=l$, we get a collection $y_{0}, \ldots, y_{k-1}, y_{k+1}, \ldots, y_{q+1}$ of $q$-simplices in $E_{q}$ such that $\partial_{i} y_{j}=\partial_{j-1} y_{i}$ for $i<j, p y_{i}=\partial_{i} b$, and $\left[y_{i}\right]=\left[x_{i}\right]$. Let

$$
y=\lambda\left(b ; y_{0}, \ldots, y_{k-1},-, y_{k+1}, \ldots, y_{q+1}\right) \in E_{q+1} \text {. }
$$

Then $\partial_{i}[y]=\left[x_{i}\right]$, and $p^{n}([y])=b$, so define

$$
\bar{\lambda}\left(b ;\left[x_{0}\right], \ldots,\left[x_{k-1}\right],-,\left[x_{k+1}\right], \ldots,\left[x_{q+1}\right]\right)=[y] .
$$

To complete the proof, we must show that $\bar{\lambda}$ satisfies the degeneracy condition

$$
\begin{aligned}
& \bar{\lambda}\left(s_{m} p^{n}\left[x^{\prime}\right] ; \partial_{0} s_{m}\left[x^{\prime}\right], \ldots, \partial_{k-1} s_{m}\left[x^{\prime}\right],-,\right. \\
&\left.\partial_{k+1} s_{m}\left[x^{\prime}\right], \ldots, \partial_{q+1} s_{m}\left[x^{\prime}\right]\right)=s_{m}\left[x^{\prime}\right]
\end{aligned}
$$

for $\left[x^{\prime}\right] \in E_{q}^{(n)}$ and $0 \leq m \leq q$. We will need some additional notation. 
Keep $l=q-n$, and let $\left[x^{\prime}\right] \in E_{q}^{(n)}$. For $I=\left(i_{0}, \ldots, i_{l-1}\right)$, an ordered l-tuple of integers $i_{j}$ satisfying (7.2), define $c_{0}(I)=\partial_{I}\left[x^{\prime}\right] \in E_{n}$.

For an ordered $(l-1)$-tuple $I=\left(i_{1}, \ldots, i_{l-1}\right)$ of integers $i_{j}$ satisfying (7.2), define

$$
c_{1}(I)=\lambda\left(\partial_{I} p^{n}\left[x^{\prime}\right] ;-, \partial_{1} \partial_{I}\left[x^{\prime}\right], \ldots, \partial_{n+1} \partial_{I}\left[x^{\prime}\right]\right) \in E_{n+1} .
$$

Since $\partial_{i} \partial_{I}\left[x^{\prime}\right] \in E_{n}$ and since $p: E \rightarrow B$ is minimal, $\partial_{0} c_{1}(I)=\partial_{0} \partial_{I}\left[x^{\prime}\right]$. Thus, $\partial_{i} c_{1}(I)=\partial_{i} \partial_{I}\left[x^{\prime}\right]=c_{0}(i, I)$ for all $i$.

Let $0<r<l$ and assume $c_{r}(I) \in E_{n+r}$ is defined for all $(l-r)$-tuples $I=\left(i_{r}, \ldots, i_{l-1}\right)$ of integers $i_{j}$ satisfying (7.2) such that $\partial_{i} c_{r}(I)=c_{r-1}(i, I)$ for all $i \neq k$ and $p c_{r}(I)=\partial_{I} p^{n}\left[x^{\prime}\right]$. Let $I=\left(i_{r+1}, \ldots, i_{l-1}\right)$ be an $(l-r-1)$ tuple of integers $i_{j}$ satisfying (7.2), and define

$$
\begin{aligned}
c_{r+1}(I)=\lambda\left(\partial_{I} p^{n}\left[x^{\prime}\right] ;\right. & c_{r}(0, I), \ldots, c_{r}(k-1, I),-, \\
& \left.c_{r}(k+1, I), \ldots, c_{r}(n+r+1, I)\right) \in E_{n+r+1} .
\end{aligned}
$$

Then $\partial_{i} c_{r+1}(I)=c_{r}(i, I)$ for all $i \neq k$, and $p c_{r+1}(I)=\partial_{I} p^{n}\left[x^{\prime}\right]$.

Finally, define

$$
c_{l}=\lambda\left(p^{n}\left[x^{\prime}\right] ; c_{l-1}(0), \ldots, c_{l-1}(k-1),-, c_{l-1}(k+1), \ldots, c_{l-1}(q)\right) \in E_{q} .
$$

Then $\partial_{i} \partial_{i_{1}} \cdots \partial_{i_{l-1}} c_{l}=\partial_{i} \partial_{i_{1}} \cdots \partial_{i_{l-1}}\left[x^{\prime}\right]$ for all $i$ and for all integers $i_{j}$ satisfying (7.2), and $p c_{l}=p^{n}\left[x^{\prime}\right]$. Thus, $\left[c_{l}\right]=\left[x^{\prime}\right]$.

Now we are ready to prove the degeneracy condition. Let $\left[x_{i}\right]=\partial_{i} s_{m}\left[x^{\prime}\right]$ and $b=s_{m} p^{n}\left[x^{\prime}\right]$, and define all the simplices $y_{\left(i_{r}, \ldots, i_{l}\right)}$ as in the construction of

$$
\bar{\lambda}\left(b ;\left[x_{0}\right], \ldots,\left[x_{k-1}\right],-,\left[x_{k+1}\right], \ldots,\left[x_{q+1}\right]\right)
$$

above. We leave it to the reader to prove by induction that

$$
y_{\left(i_{r}, \ldots, i_{l}\right)}= \begin{cases}c_{r}(I) & \text { if } \partial_{i_{r}} \cdots \partial_{i_{l}} s_{m}=\partial_{I} \text { for some } I, \\ s_{m^{\prime}} c_{r-1}\left(I^{\prime}\right) & \text { if } \partial_{i_{r}} \cdots \partial_{i_{l}} s_{m}=s_{m^{\prime}} \partial_{I^{\prime}} \text { for some } m^{\prime} \text { and } I^{\prime} .\end{cases}
$$

When $r=l$, we have $y_{i}=\partial_{i} s_{m} c_{l}$ for all $i$, so

$$
\begin{aligned}
y & =\lambda\left(s_{m} p c_{l} ; \partial_{0} s_{m} c_{l}, \ldots, \partial_{k-1} s_{m} c_{l},-, \partial_{k+1} s_{m} c_{l}, \ldots, \partial_{q+1} s_{m} c_{l}\right) \\
& =s_{m} c_{l}=s_{m}\left[x^{\prime}\right] .
\end{aligned}
$$

This proves the degeneracy condition and completes the proof of Lemma 7.1.

\section{REFERENCES}

1. R. Arens, Topologies for homeomorphism groups, Amer. J. Math. 68 (1946), 593-610.

2. A. Borel and N. Wallach, Continuous cohomology, discrete subgroups, and representations of reductive groups, Princeton Univ. Press, Princeton, N.J., 1980.

3. E. H. Brown and R. H. Szczarba, Continuous cohomology and real homotopy type, Trans. Amer. Math. Soc. 311 (1989), 57-106.

4. E. Curtis, Simplicial homotopy theory, mimeographed lecture notes, Aarhus Universitet. 
5. A. Haefliger, Feuilletages sur les variétés ouvertes, Topology 9 (1970), 183-194.

6. J. P. May, Simplicial objects in algebraic topology, Univ. of Chicago Press, Chicago, Ill., 1982.

7. G. Segal, Classifying spaces and spectral sequences, Inst. Hautes Études Sci. Publ. Math. $\mathbf{3 4}$ (1968), 105-112.

8. _ Classifying spaces related to foliations, Topology 17 (1978), 367-382.

9. N. E. Steenrod, A convenient category of topological spaces, Michigan Math. J. 14 (1967), 133-152.

10. W. T. Van Est, On the algebraic cohomology concepts in Lie groups. I, II, Nederl. Akad. Wetensch. Proc. Ser. A 58 (1955), 225-233, 286-294.

Department of Mathematics, Yale University, Box 2155 Yale Station, New Haven, CONNECTICUT 06520

Current address (8/90-6/91): The University of Maryland, European Division, APO New York 09102 